\title{
FINITE-DIFFERENCES DISCRETIZATIONS OF THE MUMFORD-SHAH FUNCTIONAL
}

\author{
Antonin Chambolle ${ }^{1}$
}

\begin{abstract}
About two years ago, Gobbino [21] gave a proof of a De Giorgi's conjecture on the approximation of the Mumford-Shah energy by means of finite-differences based non-local functionals. In this work, we introduce a discretized version of De Giorgi's approximation, that may be seen as a generalization of Blake and Zisserman's "weak membrane" energy (first introduced in the image segmentation framework). A simple adaptation of Gobbino's results allows us to compute the $\Gamma$-limit of this discrete functional as the discretization step goes to zero; this generalizes a previous work by the author on the "weak membrane" model [10]. We deduce how to design in a systematic way discrete image segmentation functionals with "less anisotropy" than Blake and Zisserman's original energy, and we show in some numerical experiments how it improves the method.

Résumé. Une conjecture récente de De Giorgi, sur l'approximation de la fonctionnelle de Mumford et Shah par des fonctionnelles non locales basées sur des différences finies, a été démontrée il y a un peu plus de deux ans par Gobbino [21]. Nous introduisons dans ce travail une version discrétisée de l'approximation de De Giorgi, que l'on peut voir comme une généralisation de l'énergie de "membrane faible" introduite par Blake et Zisserman pour la segmentation d'images. Une adaptation élémentaire des démonstrations de Gobbino permet de calculer la $\Gamma$-limite de cette approximation discrète, lorsque le pas de discrétisation tend vers zéro ; ce calcul généralise un résultat précédent de l'auteur sur l'énergie de "membrane faible" [10]. On déduit ainsi une manière de construire systématiquement des fonctionnelles de segmentation d'images "moins anisotropes" que l'énergie originale de Blake et Zisserman, et l'amélioration obtenue est illustrée par des expériences numériques.
\end{abstract}

AMS Subject Classification. 26A45, 49J45, 49Q20, 68U10.

Received: September 22, 1997. Revised: February 15, 1998.

\section{INTRODUCTION}

In winter 1995-96, De Giorgi imagined the following non-local functional, defined for any measurable function $u$ on $\mathbb{R}^{N}$,

$$
E_{\varepsilon}(u)=\iint_{\mathbb{R}^{N} \times \mathbb{R}^{N}} \frac{1}{\varepsilon} \operatorname{arctg}\left(\frac{(u(x)-u(x+\varepsilon \xi))^{2}}{\varepsilon}\right) e^{-|\xi|^{2}} d x d \xi
$$

Keywords and phrases. Free discontinuity problems, $\Gamma$-convergence, special bounded variation ( $S B V)$ functions, finite differences, image processing.

1 CEREMADE (CNRS UMR 7534), Université de Paris-Dauphine, 75775 Paris Cedex 16, France.

e-mail: Antonin.Chambolle@ceremade.dauphine.fr

(C) EDP Sciences, SMAI 1999 
as a possible approximation, as $\varepsilon$ goes to zero, of the non-convex part of the Mumford-Shah functional

$$
E(u)=\lambda \int_{\mathbb{R}^{N}}|\nabla u(x)|^{2} d x+\mu \mathcal{H}^{N-1}\left(S_{u}\right)
$$

defined on functions $u \in G S B V_{l o c}\left(\mathbb{R}^{N}\right)$ of "generalized special functions with bounded variation" (for the precise definition and main properties of this space, and more information about the Mumford-Shah functional, see Appendix A), with $S_{u}$ the "jump set" of $u$ and $\mathcal{H}^{N-1}$ the $N-1$ dimensional Hausdorff measure, and $\lambda, \mu$ two positive parameters.

One year later, Gobbino [21] gave a proof of De Giorgi's conjecture: he established that, as $\varepsilon \downarrow 0, E_{\varepsilon}$ $\Gamma$-converges to $E$ in the strong $L^{p}\left(\mathbb{R}^{N}\right)$ topology for any $1 \leq p<+\infty$, for $\lambda=\frac{\sqrt{\pi}^{N}}{2}$ and $\mu=\lambda \sqrt{\pi}$. (See Appendix B for the definition and properties of the $\Gamma$-convergence.)

Mumford and Shah's functional was introduced first in [22] as a possible continuous version of the following "weak membrane" energy, that Blake and Zisserman [7] had suggested to minimize in order to solve the image segmentation problem:

$$
\sum_{i, j} f\left(\left|u_{i+1, j}-u_{i, j}\right|^{2}\right)+f\left(\left|u_{i, j+1}-u_{i, j}\right|^{2}\right)+\left|u_{i, j}-g_{i, j}\right|^{2}
$$

where $f(x)=\alpha x \wedge \beta(\alpha, \beta>0$ and $X \wedge Y$ denotes $\min (X, Y)),\left(g_{i, j}\right)_{1 \leq i, j \leq n} \in[0,1]^{n \times n}$ is an "original" grey-level image of $n \times n$ pixels, and $\left(u_{i, j}\right)_{1 \leq i, j \leq n}$ is the output "segmented" image (here a discontinuity is supposed to occur between two adjacent pixels when the difference between the values of $u$ on each pixel is greater than $\sqrt{\beta / \alpha})$.

By minimizing this discrete functional, the aim is to rebuild from the original data $g$ a piecewise regular image $u$ (the discontinuities of $u$ corresponding to the edges in the image) that should be easier to analyze, in particular when the original is corrupted by noise (Blake and Zisserman's energy is actually derived from the free energy introduced in [19], where S. and D. Geman propose a probabilistic approach to the edge-preserving denoising of discrete images corrupted by an additive Gaussian noise). Many image reconstruction methods have been based on this kind of energies since the late 80 's, we refer for instance to [16,17], to [18] and to [6] where some variants are presented and applications are described.

In order to understand the relationship between Mumford and Shah's functional and Blake and Zisserman's, the author studied in [10] the behaviour of the discrete image segmentation problem as the pixels' size goes to zero and their number to infinity (see also [9] for the one-dimensional case) and proved the $\Gamma$-convergence of the weak membrane energy, as $n \rightarrow \infty$, to an anisotropic version of the Mumford-Shah functional, namely

$$
\alpha \int_{(0,1)^{2}}|\nabla u(x)|^{2} d x+\beta \int_{S_{u}}\left(\left|\nu_{1}\right|+\left|\nu_{2}\right|\right) d \mathcal{H}^{1}+\int_{(0,1)^{2}}|u(x)-g(x)|^{2} d x
$$

where $\nu=\left(\nu_{1}, \nu_{2}\right) \in \mathbb{S}^{1}$ is the unit normal to $S_{u}$ (defined $\mathcal{H}^{1}$-a.e.). In order to obtain this result Blake and Zisserman's energy had to be weighted and rewritten in the following way, introducing the discretization step $h=1 / n$ :

$$
h^{2} \sum_{i, j} \frac{1}{h} f\left(\frac{\left|u_{i+1, j}-u_{i, j}\right|^{2}}{h}\right)+\frac{1}{h} f\left(\frac{\left|u_{i, j+1}-u_{i, j}\right|^{2}}{h}\right)+\left|u_{i, j}-g_{i, j}^{h}\right|^{2},
$$

$g^{h}$ being some reasonable discretization of $g$ at the scale $h$.

In some of the above mentioned image processing papers it had been noticed that the results could be improved by trying to modify slightly the energy, making it "less anisotropic". This paper deals with the mathematical analysis of such variants, and gives a general solution. We explain 1) how Blake and Zisserman's energy has to 
be modified in order to approximate better the Mumford-Shah functional; 2) how this mathematical result can be exploited to actually obtain better segmentations of images.

More precisely, we rely in this paper on Gobbino's result to establish a generalization of the approximation theorem of [10] to discrete energies involving arbitrary finite differences and more general functions $f$. The practical consequences of this rather theoretical result are then described in Section 2, where we show how to design variants of the weak membrane energy, that approximate better (i.e., in a "less anisotropic" way) the Mumford-Shah functional, and show in numerical examples that they produce "nicer" segmentations.

Let $\Omega \subseteq \mathbb{R}^{N}$ be an open domain with Lipschitz boundary, and for every $h>0$ and every $u: \Omega \cap h \mathbb{Z}^{N} \rightarrow \mathbb{R}$ let

$$
F_{h}(u, \Omega)=h^{N} \sum_{\left\{\begin{array}{l}
x \in h \mathbb{Z}^{N} \\
x \in \Omega
\end{array}\right.} \sum_{\substack{\xi \in \mathbb{Z}^{N} \\
x+h \xi \in \Omega}} \frac{1}{h} f_{\xi}\left(\frac{(u(x)-u(x+h \xi))^{2}}{h}\right) \phi(\xi) \in[0,+\infty],
$$

where:

- $\phi: \mathbb{Z}^{N} \rightarrow[0,+\infty)$ is even, satisfies $\phi(0)=0, \sum_{\xi \in \mathbb{Z}^{N}}|\xi|^{2} \phi(\xi)<+\infty$, and $\phi\left(e_{i}\right)>0$ for any $i=1, \ldots, N$ where $\left(e_{i}\right)_{1 \leq i \leq N}$ is the canonical basis of $\mathbb{R}^{N}$ (in practical applications the support of $\phi$ will have to be finite and small);

- for any $\xi$ with $\phi(\xi)>0, f_{\xi}:[0,+\infty) \rightarrow[0,+\infty)$ is a non-decreasing bounded function with $f_{\xi} \equiv f_{-\xi}$, $f_{\xi}(0)=0, f_{\xi}^{\prime}(0)=\alpha_{\xi}>0$, and $\lim _{t \rightarrow+\infty} f_{\xi}(t)=\beta_{\xi}$, and we assume that $f_{\xi}$ is below (or equal to) the function $t \mapsto \alpha_{\xi} t \wedge \beta_{\xi}$. We also assume both $\sup _{\xi \in \mathbb{Z}^{N}} \alpha_{\xi}$ and $\sup _{\xi \in \mathbb{Z}^{N}} \beta_{\xi}$ are finite;

- we will adopt in the sequel the convention that any term in the sum above is zero whenever either $x$ or $x+h \xi$ is not in $\Omega$ even if we do not explicitly write these conditions under the summation signs (this convention will be adopted throughout the whole paper unless otherwise stated), as well, we'll usually write $F_{h}(u)$ instead of $F_{h}(u, \Omega)$ when not ambiguous.

Fix $p \in[1,+\infty)$, and let $\ell^{p}\left(\Omega \cap h \mathbb{Z}^{N}\right)$ be the vector space of functions $u: \Omega \cap h \mathbb{Z}^{N} \rightarrow \mathbb{R}$ such that the norm

$$
\|u\|_{p}=\left\{h^{N} \sum_{x \in \Omega \cap h \mathbb{Z}^{N}}|u(x)|^{p}\right\}^{\frac{1}{p}}
$$

is finite. In the whole paper we will always identify a function $u$ in $\ell^{p}\left(\Omega \cap h \mathbb{Z}^{N}\right)$ and the piecewise constant function in $L^{p}\left(\mathbb{R}^{N}\right)$ equal to $u(x)$ on $x+\left(-\frac{h}{2}, \frac{h}{2}\right)^{N}$ for any $x \in \Omega \cap h \mathbb{Z}^{N}$ (and to 0 elsewhere), so that $\|u\|_{p}=\|u\|_{L^{p}\left(\mathbb{R}^{N}\right)}$ and that a sentence such as " $u_{h} \in \ell^{p}\left(\Omega \cap h \mathbb{Z}^{N}\right)$ converges to $u \in L^{p}(\Omega)$ as $h \downarrow 0$ " will have a natural sense. We also set $F_{h}(u)=+\infty$ for any $u \in L^{p}(\Omega)$ that is not the restriction to $\Omega$ of the piecewise constant extension of a function in $\ell^{p}\left(\Omega \cap h \mathbb{Z}^{N}\right)$.

Let now, for any $u \in L^{p}(\Omega) \cap G S B V_{l o c}(\Omega)$,

$$
F(u)=\int_{\Omega} \sum_{\xi \in \mathbb{Z}^{N}} \phi(\xi) \alpha_{\xi}|\langle\nabla u(x), \xi\rangle|^{2} d x+\int_{S_{u}} \sum_{\xi \in \mathbb{Z}^{N}} \phi(\xi) \beta_{\xi}\left|\left\langle\nu_{u}(x), \xi\right\rangle\right| d \mathcal{H}^{N-1}(x) \in[0,+\infty],
$$

and set $F(u)=+\infty$ if $u \in L^{p}(\Omega) \backslash G S B V_{l o c}(\Omega)$. We will also denote sometimes

$$
F(u, B)=\int_{B} \sum_{\xi \in \mathbb{Z}^{N}} \phi(\xi) \alpha_{\xi}|\langle\nabla u(x), \xi\rangle|^{2} d x+\int_{B \cap S_{u}} \sum_{\xi \in \mathbb{Z}^{N}} \phi(\xi) \beta_{\xi}\left|\left\langle\nu_{u}(x), \xi\right\rangle\right| d \mathcal{H}^{N-1}(x)
$$

when $B \subseteq \Omega$ is a Borel set. An adaptation of Gobbino's results leads to the following theorem. 
Theorem 1. $F_{h} \Gamma$-converges to $F$ as $h \downarrow 0$ in the strong topology of $L^{p}(\Omega)$, for any $p \in[1,+\infty)$.

As a corollary, we also prove

Theorem 2. Let $p \in[1,+\infty), g \in L^{p}(\Omega) \cap L^{\infty}(\Omega)$, and for any $h>0$ let $u_{h}$ be a minimizer over $\ell^{p}\left(\Omega \cap h \mathbb{Z}^{N}\right)$ of

$$
F_{h}(u)+\int_{\Omega}|u(x)-g(x)|^{p} d x
$$

(or, equivalently, of

$$
F_{h}(u)+\left(\left\|u-g^{h}\right\|_{p}\right)^{p}
$$

where $g^{h} \in \ell^{p}\left(\Omega \cap h \mathbb{Z}^{N}\right)$ is a suitable discretization of $g$ at scale $h$, with $g^{h} \rightarrow g$ in $L^{p}(\Omega)$ as $h \downarrow 0$ and $\left\|g^{h}\right\|_{\infty} \leq\|g\|_{\infty}$ for all $\left.h\right)$. Then $\left(u_{h}\right)$ is relatively compact in $L^{p}(\Omega)$ and if some subsequence $u_{h_{j}}$ goes to $u$ as $j \rightarrow \infty, u \in S B V_{l o c}(\Omega) \cap L^{p}(\Omega)$ is a minimizer of

$$
F(u)+\int_{\Omega}|u(x)-g(x)|^{p} d x .
$$

Remark 1. In [10], Theorem 2 is proved for $p=2, N=2, \Omega=(0,1) \times(0,1), \phi \equiv 0$ on $\mathbb{Z}^{2}$ except $\phi(0,1)=$ $\phi(1,0)=\phi(0,-1)=\phi(-1,0)=1 / 2$, and $f_{\xi}(t)=\alpha t \wedge \beta(\alpha, \beta>0)$, so that

$$
F(u)=\alpha \int_{\Omega}|\nabla u(x)|^{2} d x+\beta \int_{S_{u}}\left\|\nu_{u}(x)\right\|_{1} d \mathcal{H}^{1}(x),
$$

where $\|x\|_{1}=\left|x_{1}\right|+\left|x_{2}\right|$ for any $x \in \mathbb{R}^{2}$.

Remark 2. A few other discrete approximations of the Mumford-Shah functional have been proposed. G. Bellettini and A. Coscia have established the $\Gamma$-convergence of finite elements based approximating functionals, to the original isotropic functional $E$. Their result is based on the discretization of L. Ambrosio and Tortorelli's approximation $[4,5]$, in which the discontinuity set is represented by an auxiliary function $v$ that in some sense concentrates near the set as the approximation comes closer to the solution. In [11], Dal Maso and the author have proposed an alternate finite elements method for approximating the Mumford-Shah functional $E$.

Remark 3. The condition $\phi\left(e_{i}\right)>0$ for $i=1, \ldots, N$ is necessary only for the coercivity, i.e. to establish Lemma 1 and Theorem 2. This is important in practical applications for the stability of the numerical schemes. For the $\Gamma$-convergence it would be sufficient to assume that $\phi\left(\xi_{i}\right)>0, i=1, \ldots, N$ for some basis $\left(\xi_{i}\right)_{1 \leq i \leq N} \in$ $\mathbb{Z}^{N \times N}$ of $\mathbb{R}^{N}$.

In the next section we describe a numerical implementation of the minimization problem (3), and show how the choice of $\phi, \alpha_{\xi}$ and $\beta_{\xi}$ influence the results. Then, we prove Theorem 1 (explaining how Gobbino's proofs can be adapted to this case) and deduce Theorem 2.

\section{Applications}

The version of Gobbino's result we present in this paper has interesting consequences, that will be clear in the few numerical examples (in $\Omega=(0,1) \times(0,1) \subset \mathbb{R}^{2}$, and with $p=2$ ) we will show later on.

\subsection{An iterative procedure for minimizing (3)}

Before showing the examples, we first quickly describe a standard procedure for minimizing energies such as (3). Of course we do not pretend to compute an exact minimizer of the energy, since the high non-convexity of the problem does not allow this. However, the iterative algorithm we describe gives satisfactory results. 
A variant has been successfully implemented in the case of the approximation of [11] (see [8]). Many other similar implementations have been made for solving image reconstruction problems (see for instance [6,23], and the pioneering work [18] by Geman and Reynolds).

We assume $\Omega$ is bounded so that the discrete problem is finite-dimensional for every fixed $h>0$ (in the applications $\Omega$ will be a rectangle). The non-convexity in the energy $F_{h}$ comes from the non-convexity of the functions $f_{\xi}, \xi \in \mathbb{Z}^{N}$. In order to simplify the computations we will assume that the $f_{\xi}$ are all identical, up to a rescaling:

$$
f_{\xi}(t)=\beta_{\xi} f\left(\frac{\alpha_{\xi}}{\beta_{\xi}} t\right)
$$

for all $\xi \in \mathbb{Z}^{\mathbb{N}}$ (with $\phi(\xi)>0$ ) and $t \geq 0$. The function $f$ is nondecreasing, and satisfies $f(0)=0, f(+\infty)=1$, and $f^{\prime}(0)=1$. We will assume as well that it is concave, and differentiable. Thus, $-f$ is convex (we extend it with the value $+\infty$ on $\{t<0\}$ ), and lower semi-continuous. Let

$$
\psi(-v)=\sup _{t \in \mathbb{R}} t v-(-f)(t)=(-f)^{*}(v)
$$

be the Legendre-Fenchel transform of $-f$, by a classical result $(-f)^{* *}=-f$ so that

$$
-f(t)=\sup _{v \in \mathbb{R}} t v-\psi(-v)=-\inf _{v \in \mathbb{R}} t v+\psi(v) .
$$

It is well-known that the first sup in this equation is attained at $v$ such that $t \in \partial(-f)^{*}(v)$ (the subdifferential of $(-f)^{*}$ at $\left.t\right)$, and that this is equivalent to $v \in \partial(-f)(t)$, and since $\partial(-f)(t)=\left\{-f^{\prime}(t)\right\}$ for $t>0$ and $(-\infty,-1]$ for $t=0$ we deduce that the sup is reached at some $v \in[-1,0]$ (since for $t=0$ we check that $(-f)^{*}(-1)=0$ and thus the sup is reached at $v=-1$ ). Hence

$$
f(t)=\min _{v \in[0,1]} t v+\psi(v)
$$

and the min is reached for $v=f^{\prime}(t)$. We may therefore rewrite $F_{h}$ in the following way:

$$
F_{h}(u)=\min _{v(\cdot, \cdot)} F_{h}(u, v)
$$

for $v:\left(\Omega \cap h \mathbb{Z}^{N}\right) \times\left(\Omega \cap h \mathbb{Z}^{N}\right) \rightarrow[0,1]$ and

$$
F_{h}(u, v)=h^{N} \sum_{x \in h \mathbb{Z}^{N}} \sum_{\xi \in \mathbb{Z}^{N}}\left\{\alpha_{\xi} v(x, x+h \xi)\left|\frac{u(x)-u(x+h \xi)}{h}\right|^{2}+\beta_{\xi} \frac{\psi(v(x, x+h \xi))}{h}\right\} \phi(\xi) .
$$

The algorithm consists in minimizing alternatively $F_{h}(u, v)+\left\|u-g^{h}\right\|_{2}^{2}$ with respect to $u$ and $v$. The minimization with respect to $v$ is straightforward, since it just consists in computing for each $x, y \in \Omega \cap h \mathbb{Z}^{N}$

$$
v(x, y)=f^{\prime}\left(\alpha_{\xi} \frac{(u(x)-u(y))^{2}}{\beta_{\xi} h}\right),
$$

with $\xi= \pm(y-x) / h$. The minimization with respect to $u$ is also a simple (linear) problem, since the energy is convex and quadratic with respect to $u$. Of course there is no way of knowing whether the algorithm converges to a solution or not, what is certain is that the energy decreases and goes to some critical level, while the function $u$ converges to either a critical point or, if it exists, a continuum of critical points. Notice that if $f$ is strictly increasing, $v$ is everywhere strictly positive. 
In the applications shown in this paper we considered $f(t)=\frac{2}{\pi} \operatorname{arctg} \frac{\pi x}{2}$, so that

$$
f^{\prime}(t)=\frac{1}{1+\frac{\pi^{2} x^{2}}{4}}
$$

Notice that one never has to compute explicitly the position of the edges during the minimization. Once a minimizer of the energy has been found, it is possible to extract the edges out of the segmented image by standard algorithms (using Canny's or more sophisticated edge detectors, with a very narrow kernel since the images on which the edges have to be found are piecewise smooth). The value of the auxiliary function $v$ is also a good indicator for the position of the edges (it is "large" on the edges and close to zero everywhere else), and should be taken into account. An elementary method may be for instance to consider the zero-crossings of the (discretized) operator $d^{2} u(\nabla u, \nabla u)$ in the regions where $v$ is large.

\subsection{Anisotropy of the length term}

In this section $n$ will be an integer $(n>1)$, we will set $h=1 / n$ and the functions $u$ and $g^{h}$ (defined on $\left.[0,1) \times[0,1) \cap h \mathbb{Z}^{2}\right)$ will be denoted as $n \times n$ matrices $\left(u_{i, j}\right)_{0 \leq i, j<n}$ and $\left(g_{i, j}^{h}\right)_{0 \leq i, j<n}$. We will compare the two following cases

$$
E_{n}^{1}(u)=h^{2} \sum_{i, j} \frac{\beta_{1}}{h} f\left(\alpha_{1} \frac{\left|u_{i+1, j}-u_{i, j}\right|^{2}}{\beta_{1} h}\right)+\frac{\beta_{1}}{h} f\left(\alpha_{1} \frac{\left|u_{i, j+1}-u_{i, j}\right|^{2}}{\beta_{1} h}\right)+\left|u_{i, j}-g_{i, j}^{h}\right|^{2},
$$

(i.e., some regularization of Blake and Zisserman's "weak membrane" energy) and

$$
\begin{aligned}
E_{n}^{2}(u)= & h^{2} \sum_{i, j} \frac{\beta_{2}}{h} f\left(\alpha_{2} \frac{\left|u_{i+1, j}-u_{i, j}\right|^{2}}{\beta_{2} h}\right)+\frac{\beta_{2}}{h} f\left(\alpha_{2} \frac{\left|u_{i, j+1}-u_{i, j}\right|^{2}}{\beta_{2} h}\right) \\
& +\frac{\beta_{2}^{\prime}}{h} f\left(\alpha_{2}^{\prime} \frac{\left|u_{i+1, j+1}-u_{i, j}\right|^{2}}{\beta_{2}^{\prime} h}\right)+\frac{\beta_{2}^{\prime}}{h} f\left(\alpha_{2}^{\prime} \frac{\left|u_{i-1, j+1}-u_{i, j}\right|^{2}}{\beta_{2}^{\prime} h}\right)+\left|u_{i, j}-g_{i, j}^{h}\right|^{2}
\end{aligned}
$$

By Theorem 2, the limit points of the minimizers of $E_{n}^{1}$ and $E_{n}^{2}$, as $n \rightarrow \infty$, will be minimizers of respectively

$$
E_{\infty}^{1}(u)=\lambda_{1} \int_{\Omega}|\nabla u(x)|^{2} d x+\mu_{1} \Lambda_{1}\left(S_{u}\right)+\int_{\Omega}|u(x)-g(x)|^{2} d x
$$

and

$$
E_{\infty}^{2}(u)=\lambda_{2} \int_{\Omega}|\nabla u(x)|^{2} d x+\mu_{2} \Lambda_{2}\left(S_{u}\right)+\int_{\Omega}|u(x)-g(x)|^{2} d x,
$$

(for $u \in L^{2}(\Omega) \cap G S B V(\Omega)$, and $+\infty$ otherwise) with $\Omega=(0,1) \times(0,1), \lambda_{1}=\alpha_{1}, \lambda_{2}=\alpha_{2}+2 \alpha_{2}^{\prime}$, and

$$
\begin{aligned}
& \mu_{1} \Lambda_{1}\left(S_{u}\right)=\int_{S_{u}} \beta_{1}\left(\left|\nu_{1}(x)\right|+\left|\nu_{2}(x)\right|\right) d \mathcal{H}^{1}(x), \\
& \mu_{2} \Lambda_{2}\left(S_{u}\right)=\int_{S_{u}} \beta_{2}\left(\left|\nu_{1}(x)\right|+\left|\nu_{2}(x)\right|\right)+\beta_{2}^{\prime}\left(\left|\nu_{1}(x)-\nu_{2}(x)\right|+\left|\nu_{1}(x)+\nu_{2}(x)\right|\right) d \mathcal{H}^{1}(x),
\end{aligned}
$$

where $\left(\nu_{1}(x), \nu_{2}(x)\right)$ is the normal vector to $S_{u}$ at $x$. Simple computations show that $\beta_{2}^{\prime}=\beta_{2} / \sqrt{2}$ is an optimal choice, since it minimizes the ratio of the length $\Lambda_{2}$ of the longest (with length $\Lambda_{2}$ ) vector in $\mathbb{S}^{1}$ over the length 

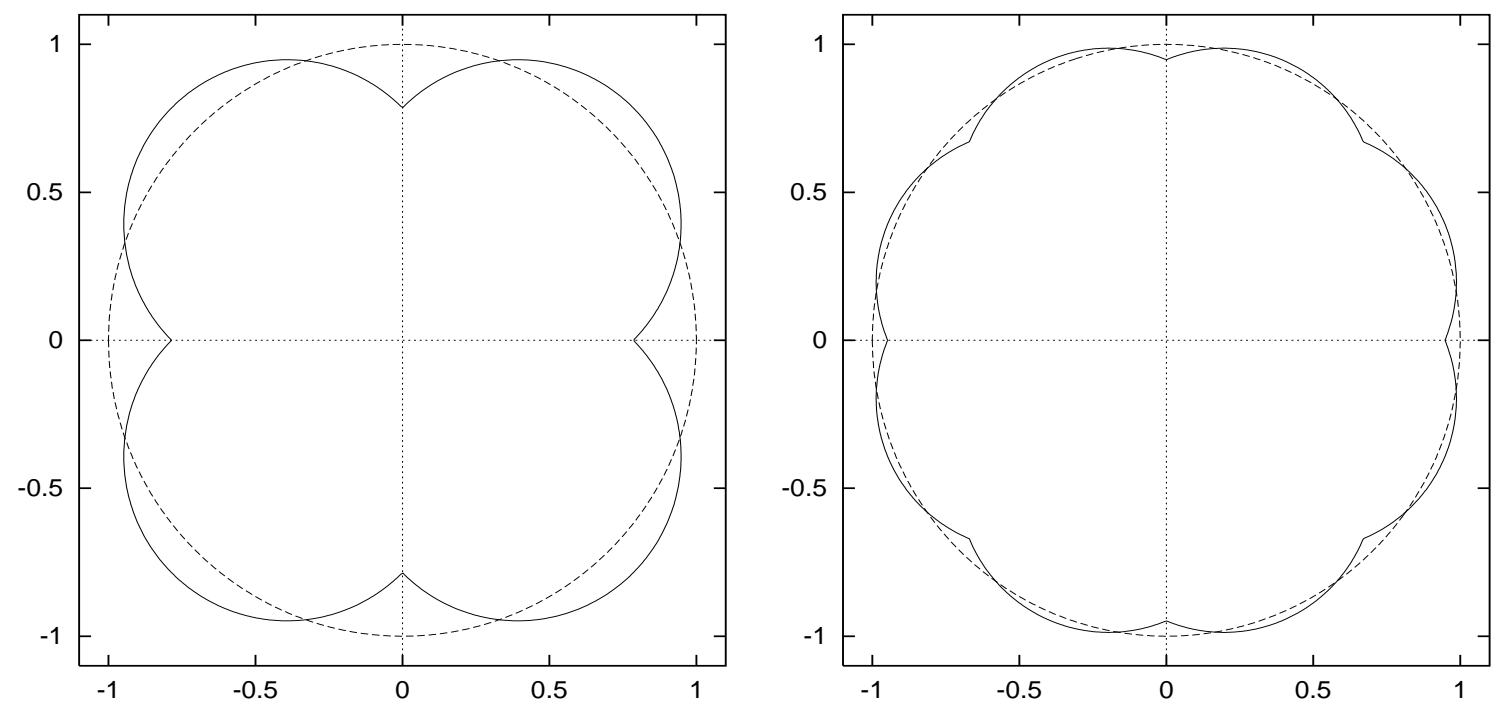

FiguRE 1. The solid line represents the length of a unit vector, as a function of the angle. Left: for $\Lambda_{1}$, right: for $\Lambda_{2}$. The dashed line is the unit circle.

of the shortest. For any rectifiable 1 -set $E \subset \mathbb{R}^{2}$ with normal vector $\left(\nu_{1}, \nu_{2}\right)$ at $x$ we define the lengths

$$
\Lambda_{1}(E)=\frac{\pi}{4} \int_{E}\left(\left|\nu_{1}(x)\right|+\left|\nu_{2}(x)\right|\right) d \mathcal{H}^{1}(x)
$$

and

$$
\Lambda_{2}(E)=\frac{\pi}{8} \int_{E}\left(\left|\nu_{1}(x)\right|+\left|\nu_{2}(x)\right|+\frac{\left|\nu_{1}(x)-\nu_{2}(x)\right|+\left|\nu_{1}(x)+\nu_{2}(x)\right|}{\sqrt{2}}\right) d \mathcal{H}^{1}(x) .
$$

(Notice that $\Lambda_{2}=\left(\Lambda_{1}+\Lambda_{1} \circ R_{\frac{\pi}{4}}\right) / 2$ where $R_{\frac{\pi}{4}}$ is the rotation of angle $\pi / 4$ in $\mathbb{R}^{2}$.) The choice of the parameters $\pi / 4$ and $\pi / 8$ is made in order to ensure that a "random" set of lines has in average the same length $\Lambda_{1}$ and $\Lambda_{2}$ (and Euclidean length), in other words, the unit circle has length $2 \pi$ in both cases. This is of course not the only possible choice. For instance, one could prefer to parametrize these lengths in such a way that the error (with respect to the Euclidean length) $\Lambda_{i}\left(e_{\max }\right)-1$ on the measure of the longest (for $\left.\Lambda_{i}\right)$ vector $e_{\max } \in \mathbb{S}^{1}$ is equal to the error $1-\Lambda_{i}\left(e_{\min }\right)$ on the measure of the shortest vector. In this case, one should choose as parameters $\frac{2}{1+\sqrt{2}}$ instead of $\pi / 4$ for $\Lambda_{1}$ and $\frac{2}{1+\sqrt{2}+\sqrt{4+2 \sqrt{2}}}$ instead of $\pi / 8$ for $\Lambda_{2}$.

With the choice we made, we get that $\mu_{1}=4 \beta_{1} / \pi$ and $\mu_{2}=8 \beta_{2} / \pi$. In both cases the limit energy is anisotropic, what is interesting is that the second length $\Lambda_{2}$ is far "less anisotropic" than the first length $\Lambda_{1}$. As a matter of fact, the longest vector in $\mathbb{S}^{1}$ for $\Lambda_{1}$ is about $41.4 \%$ longer than the shortest (the ratio is $\sqrt{2}$ ) while it is only $8.2 \%$ longer for the length $\Lambda_{2}$ (the ratio is $2 \sqrt{2} \cos \frac{\pi}{8} /(1+\sqrt{2})=\sqrt{4+2 \sqrt{2}} /(1+\sqrt{2})$ ). The difference of anisotropy of both lengths is striking on figure 1 .

\subsection{Numerical experiments}

We show here a few experiments, so that the reader can see for himself the difference of behaviour of the lengths $\Lambda_{1}$ and $\Lambda_{2}$. Notice that in all of our comparisons we of course always choose $\lambda_{1}=\lambda_{2}$ and $\mu_{1}=\mu_{2}$. In Figure 3 and Figure 4 (see original pictures in Fig. 2), one notices that the edges are usually nicer when length $\Lambda_{2}$ is used, whereas images obtained by minimization of energy $E_{n}^{1}$ are more "blocky". Notice in particular 

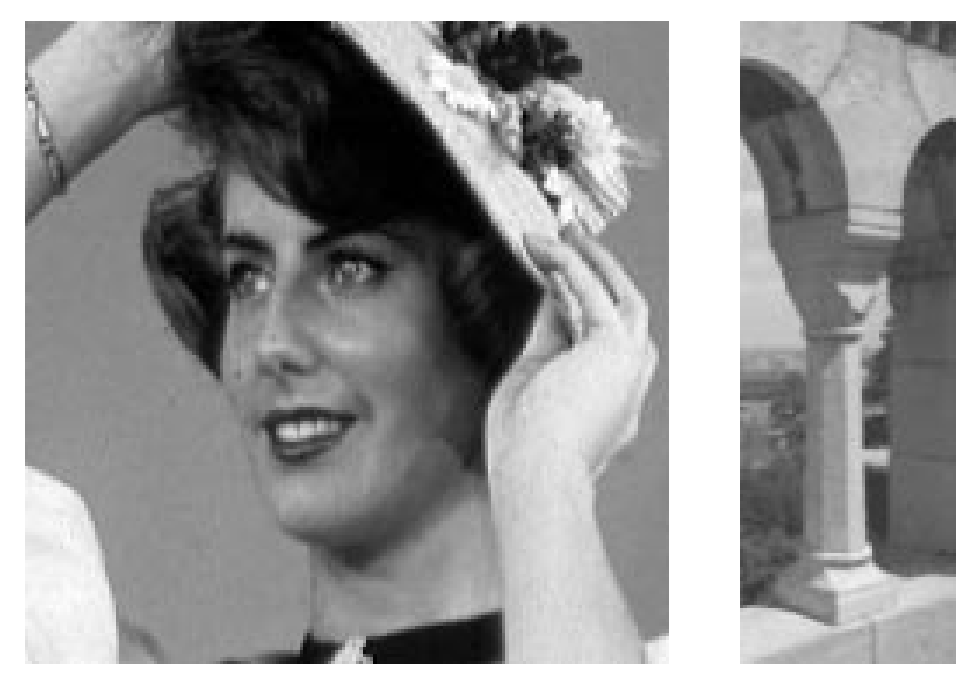

Figure 2. Original images for the next examples.
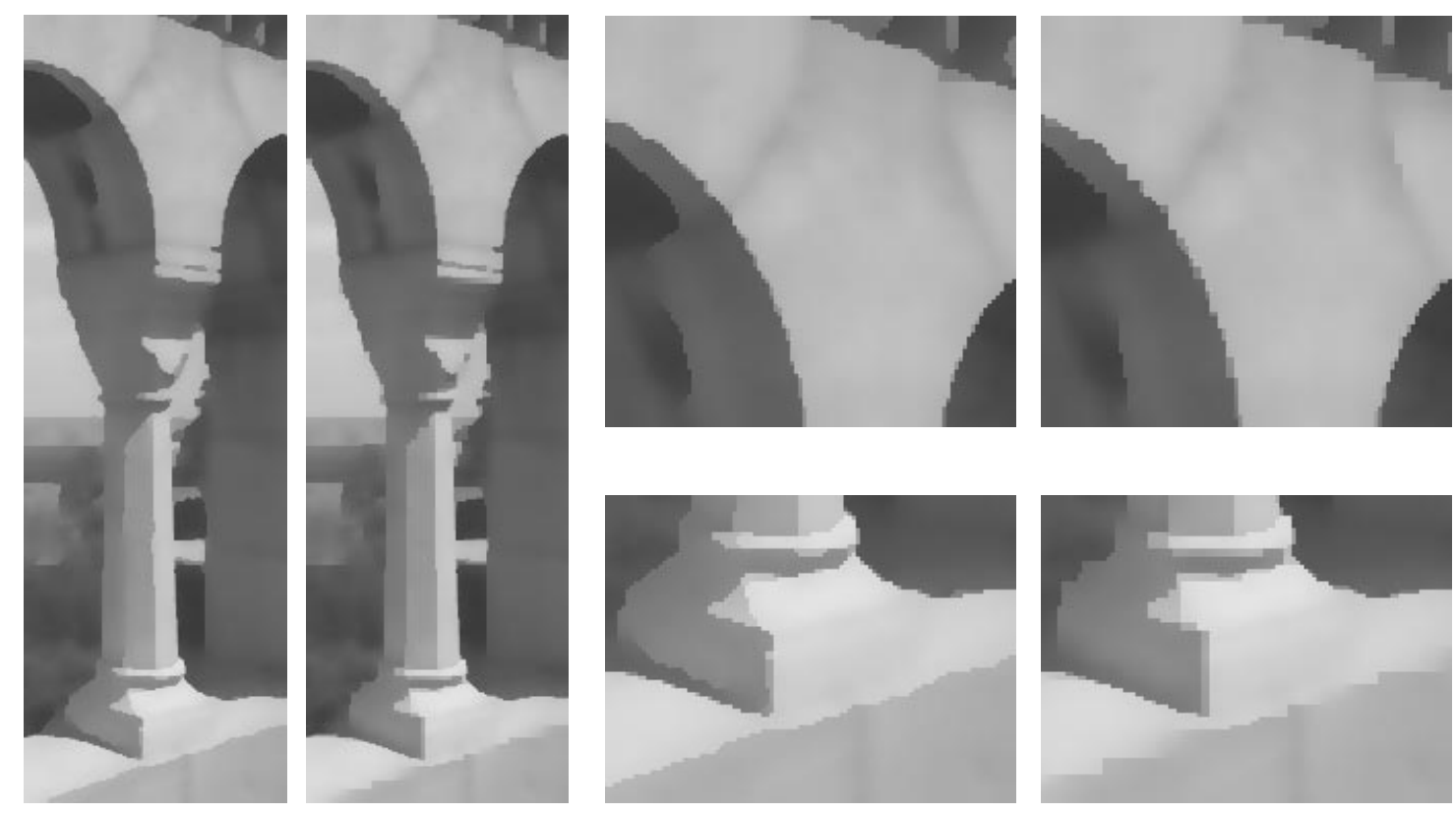

Figure 3 . The segmented column. Left, by minimizing energy $E_{n}^{2}$, then with energy $E_{n}^{1}$. The details are presented in the same order.

the diagonal line at the bottom of the image. However, the vertical edges on the column (Fig. 3, second picture) are nicer with energy $E_{n}^{1}$. The reason is clear: these edges are vertical, and the vertical and horizontal lines have a much lower costs than lines with other orientations with this energy.

In Figures 4 and 5 the results are similar: the edges look much nicer when energy $E_{n}^{2}$ is minimized. The other two figures show segmentations in presence of noise. In the two segmentations of the disk, the total length of the edges found was $6.56 \times R$ with energy $E_{n}^{2}$ and $6.40 \times R$ with $E_{n}^{1}$. These lengths are slightly overestimated 

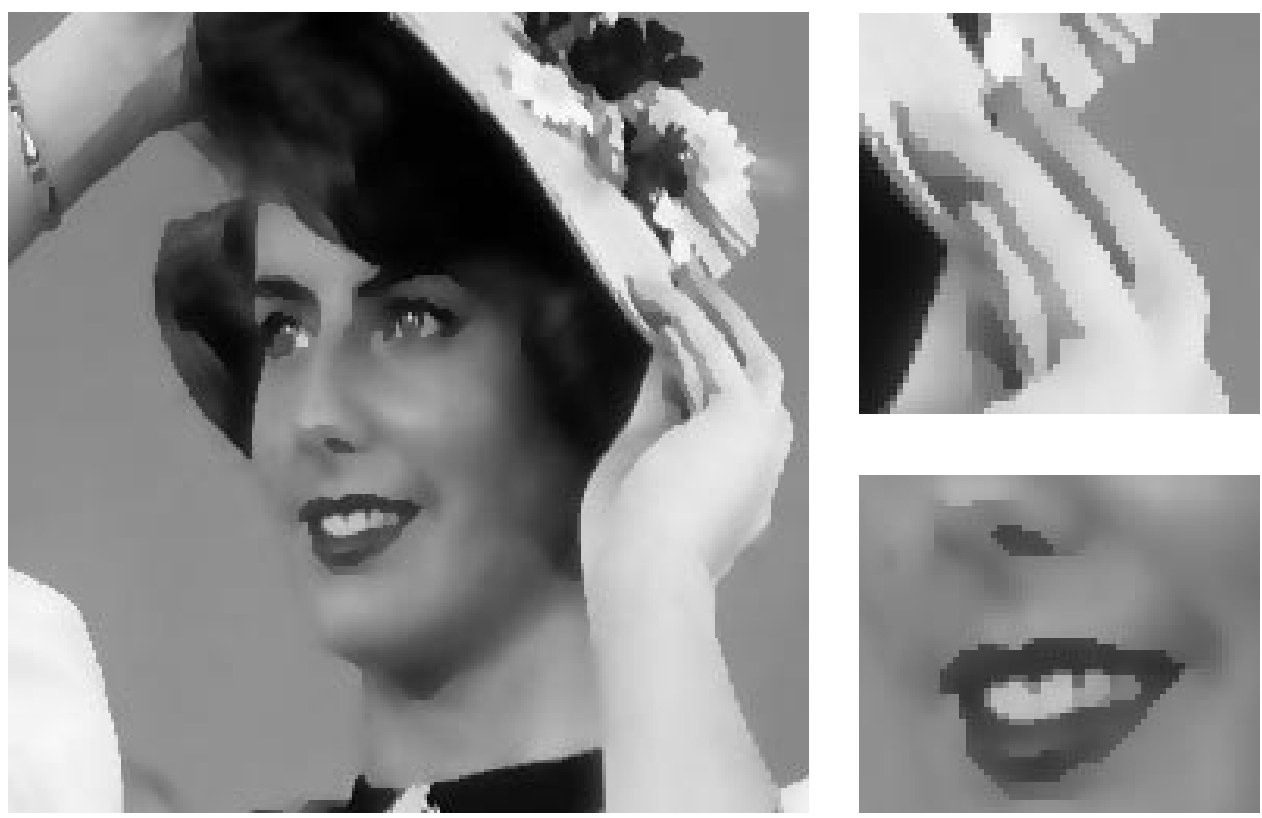

Figure 4. Segmented lady with energy $E_{n}^{2}$ and two details.
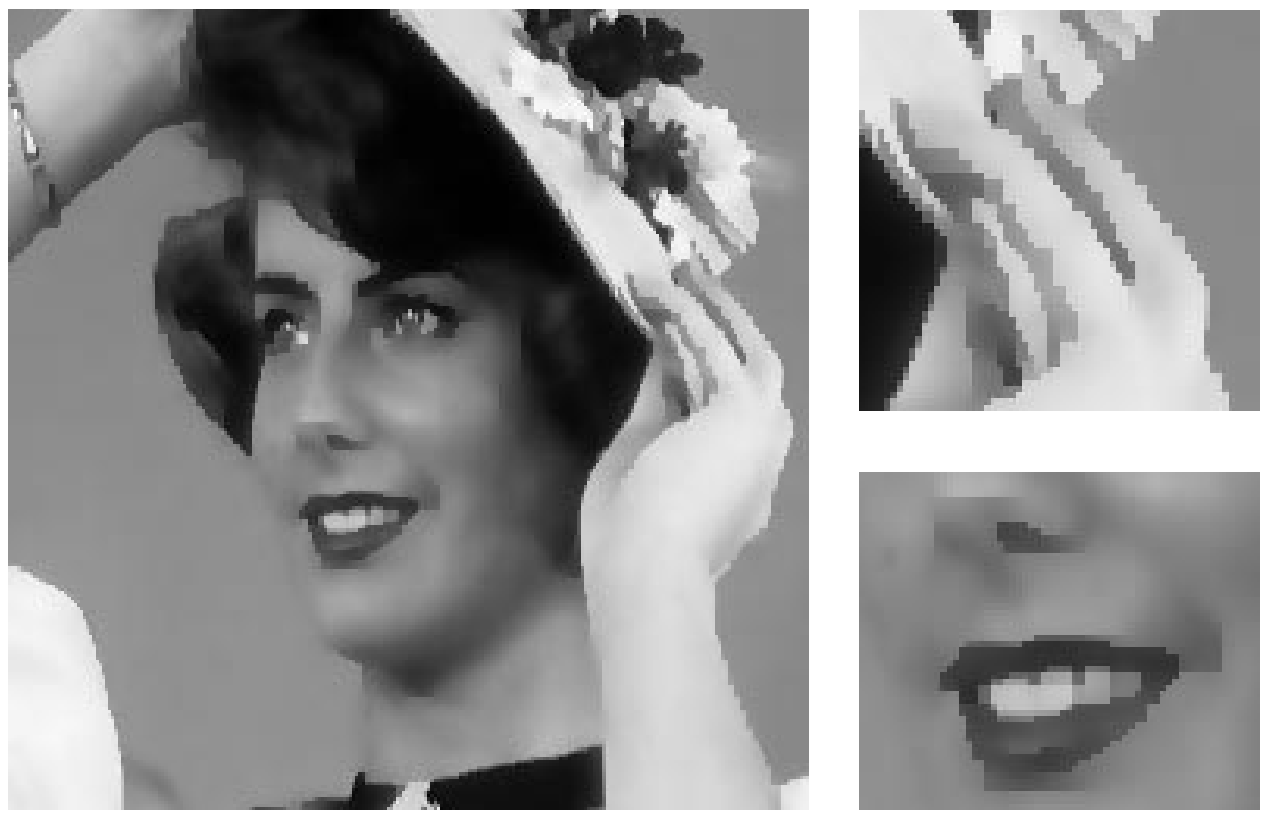

Figure 5. Segmented lady with energy $E_{n}^{1}$ and two details.

because a few spurious edges were found, and also because of some oscillation of the boundary, that is due to the noise. Again in Figure 7 the result is more blocky with energy $E_{n}^{1}$. 

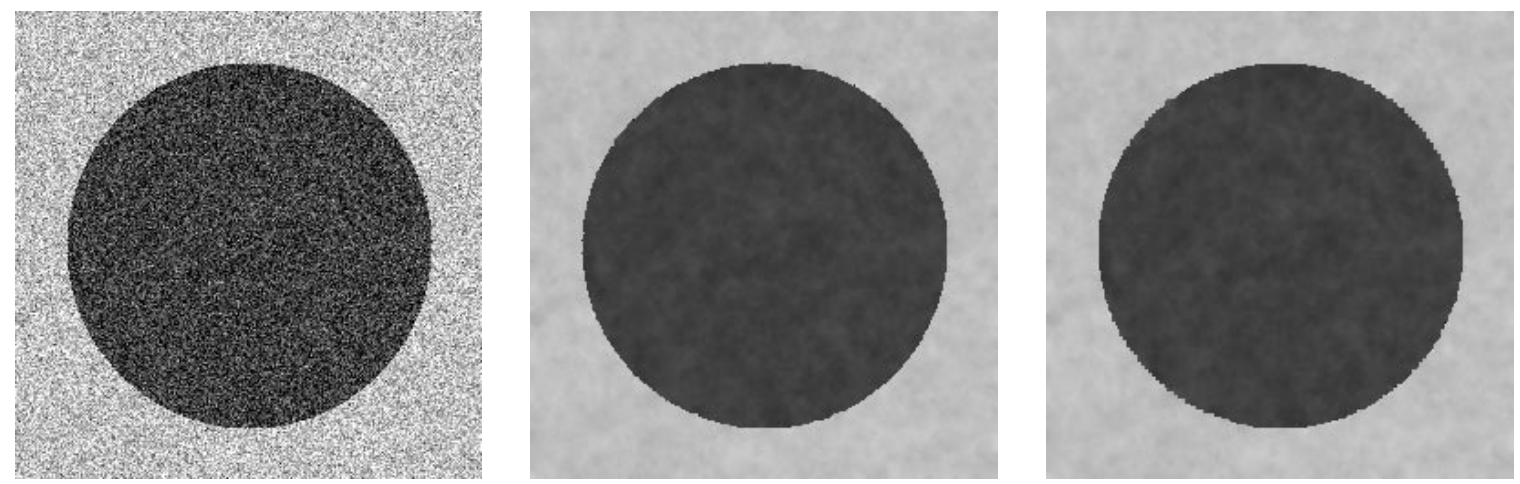

Figure 6. The noisy disk (grey level values 64 (disk) and 192 (background), std. dev. of noise 40). Middle, the segmented disk with energy $E_{n}^{2}$. Right, with $E_{n}^{1}$.
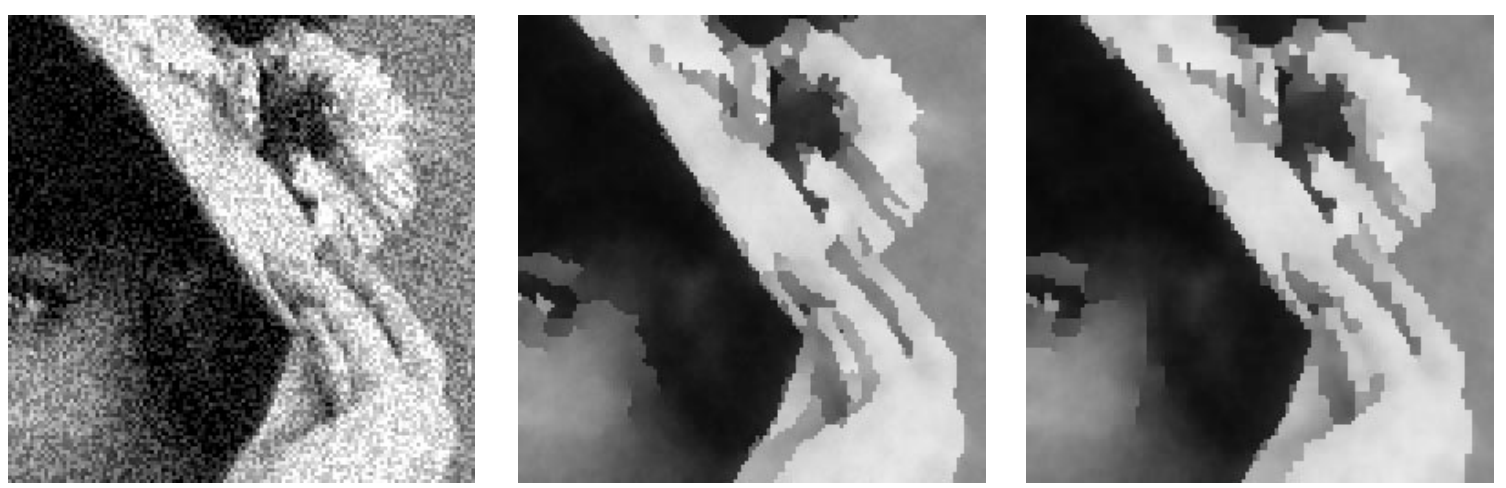

Figure 7. A noisy image (std. dev.=25 for values between 0 and 255) and the segmented outputs, by minimizing $E_{n}^{2}$ (middle) and $E_{n}^{1}$ (right).

In the last two experiments we show we used another energy, namely,

$$
\begin{aligned}
E_{n}^{3}(u)= & h^{2} \sum_{i, j} \frac{\beta_{3}}{h} f\left(\alpha_{3} \frac{\left|u_{i+1, j}-u_{i, j}\right|^{2}}{\beta_{3} h}\right)+\frac{\beta_{3}}{h} f\left(\alpha_{3} \frac{\left|u_{i, j+1}-u_{i, j}\right|^{2}}{\beta_{3} h}\right) \\
& +\frac{\beta_{3}^{\prime}}{h} f\left(\alpha_{3}^{\prime} \frac{\left|u_{i \pm 1, j+1}-u_{i, j}\right|^{2}}{\beta_{3}^{\prime} h}\right)+\frac{\beta_{3}^{\prime \prime}}{h} f\left(\alpha_{3}^{\prime \prime} \frac{\left|u_{i+2, j \pm 1}-u_{i, j}\right|^{2}}{\beta_{3}^{\prime \prime} h}\right) \\
& +\frac{\beta_{3}^{\prime \prime}}{h} f\left(\alpha_{3}^{\prime \prime} \frac{\left|u_{i \pm 1, j+2}-u_{i, j}\right|^{2}}{\beta_{3}^{\prime \prime} h}\right)+\left|u_{i, j}-g_{i, j}^{h}\right|^{2} .
\end{aligned}
$$

We choose $\beta_{3}^{\prime}=\beta_{3} / \sqrt{2}$ and $\beta_{3}^{\prime \prime}=\beta_{3} / \sqrt{5}$. Now, as $n \rightarrow \infty$, the limit points of the minimizers of $E_{n}^{3}$ minimize

$$
E_{\infty}^{3}(u)=\lambda_{3} \int_{\Omega}|\nabla u(x)|^{2} d x+\mu_{3} \Lambda_{3}\left(S_{u}\right)+\int_{\Omega}|u(x)-g(x)|^{2} d x
$$




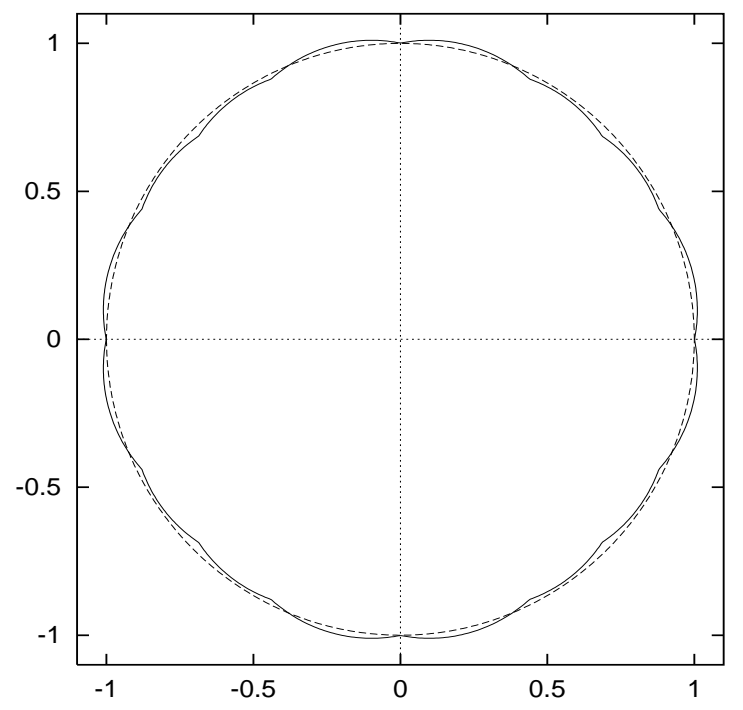

Figure 8. Same as Figure 1, this time for $\Lambda_{3}$.

with $\lambda_{3}=\alpha_{3}+2 \alpha_{3}^{\prime}+10 \alpha_{3}^{\prime \prime}$,

$$
\begin{aligned}
\Lambda_{3}(E)= & \frac{\pi}{16} \int_{E}\left(\left|\nu_{1}(x)\right|+\left|\nu_{2}(x)\right|+\frac{\left|\nu_{1}(x)-\nu_{2}(x)\right|+\left|\nu_{1}(x)+\nu_{2}(x)\right|}{\sqrt{2}}\right. \\
& \left.+\frac{\left|2 \nu_{1}(x)-\nu_{2}(x)\right|+\left|\nu_{1}(x)+2 \nu_{2}(x)\right|+\left|2 \nu_{1}(x)+\nu_{2}(x)\right|+\left|\nu_{1}(x)-2 \nu_{2}(x)\right|}{\sqrt{5}}\right) d \mathcal{H}^{1}(x)
\end{aligned}
$$

(this time $\Lambda_{3}=\left(\Lambda_{1}+\Lambda_{1} \circ R_{\frac{\pi}{4}}+\Lambda_{1} \circ R_{\theta}+\Lambda_{1} \circ R_{-\theta}\right) / 4$ with $\theta=\operatorname{arctg} 2$ ), and $\mu_{3}=16 \beta_{3} / \pi$. Figure 8 illustrates how "isotropic" the measure $\Lambda_{3}$ is, and Figures 9 and 10 show examples. (Now, the length of the longest vector in $\mathbb{S}^{1}$ is about $5.0 \%$ greater than the length of the shortest.) The results look slightly better than the ones obtained with energy $E_{n}^{2}$, however, the computational cost is quite higher.

\section{Proof of Theorem 1 And Theorem 2}

This section is entirely devoted to the proof of the theoretical results. We rely essentially on Gobbino's work [21], but since (little) adaptations are necessary in the case we present, and since, moreover, there is a very slight difference in our proof (which avoids the use of Gobbino's technical Lemmas 3.1 and 3.2 in [21]), we will give quite a few details. We let for any $u \in L^{p}(\Omega)$

$$
F^{\prime}(u)=\left(\Gamma-\liminf _{h \downarrow 0} F_{h}\right)(u)
$$

and

$$
F^{\prime \prime}(u)=\left(\Gamma-\limsup _{h \downarrow 0} F_{h}\right)(u) .
$$

In the next Section 3.1 we will prove a preliminary lemma that will be helpful in the sequel. Then, the aim of the following two Sections 3.2 and 3.3 will be to prove Theorem 1, i.e., to prove that $F^{\prime}(u) \geq F(u)$ and $F^{\prime \prime}(u) \leq F(u)$ for all $u \in L^{p}(\Omega)$. Eventually in Section 3.4, we will prove Theorem 2. 

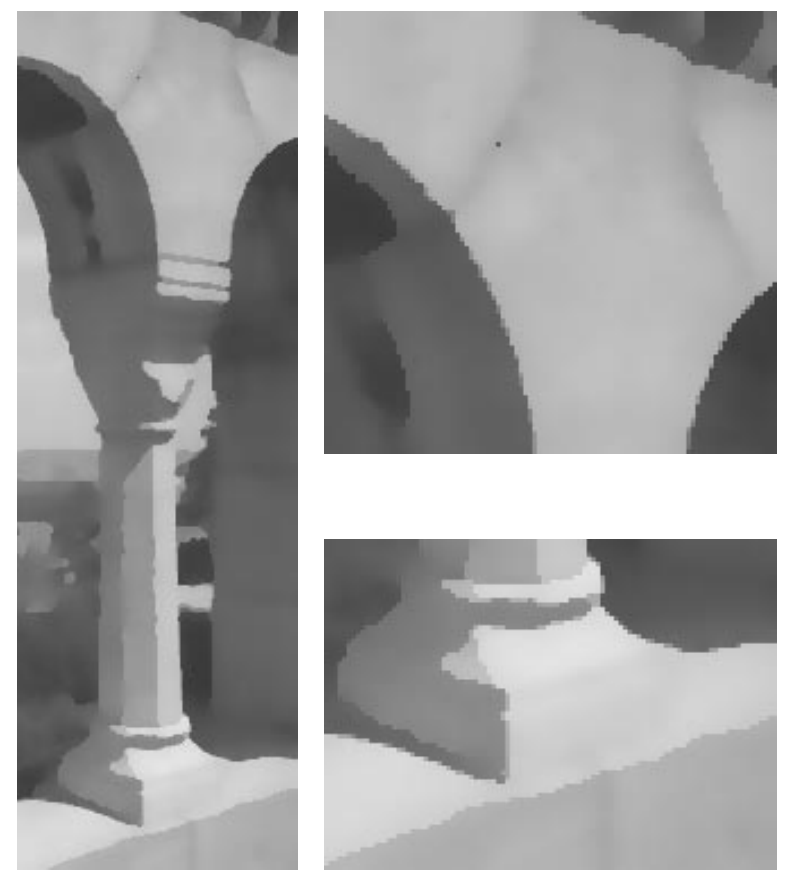

Figure 9. Segmentation with energy $E_{n}^{3}$ (the column).
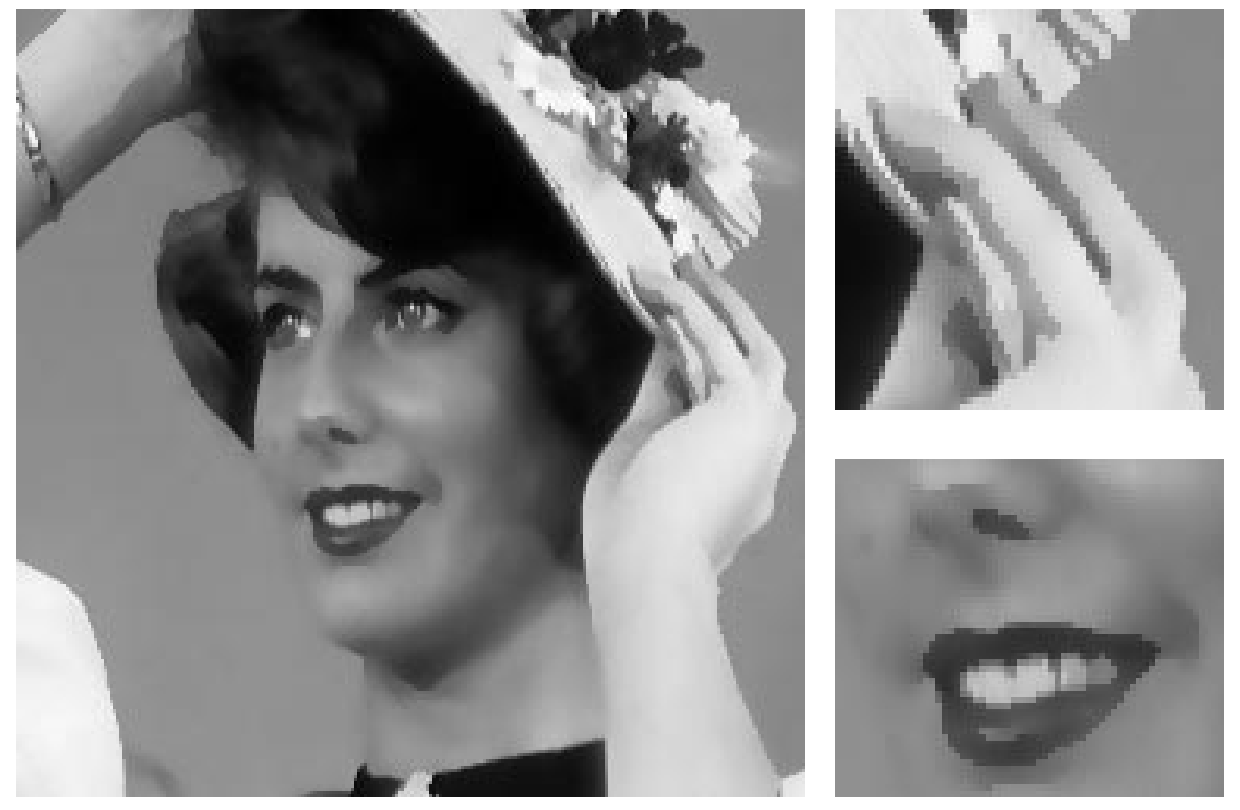

Figure 10. Segmentation with energy $E_{n}^{3}$ (the lady). 


\subsection{A compactness lemma}

The lemma we show in this section will be needed to establish Theorem 2, but it will also give some a priori information on the regularity of functions $u \in L^{p}(\Omega)$ such that $F^{\prime}(u)<+\infty$.

Lemma 1. Let $h_{j} \downarrow 0$ and $u_{h_{j}} \in \ell^{p}\left(\Omega \cap h_{j} \mathbb{Z}^{N}\right)$ such that

$$
\sup _{j} F_{h_{j}}\left(u_{h_{j}}\right)<+\infty \text { and } \sup _{j}\left\|u_{h_{j}}\right\|_{\infty}<+\infty .
$$

Then there exist a subsequence (not relabelled) $u_{h_{j}}$ and $u \in S B V_{l o c}(\Omega)$ such that

$$
u_{h_{j}}(x) \rightarrow u(x) \text { a.e. in } \Omega
$$

as $j$ goes to infinity, and

$$
\int_{\Omega}|\nabla u(x)|^{2} d x+\mathcal{H}^{N-1}\left(S_{u}\right)<+\infty
$$

Proof. In order to simplify the notations we drop the subscript $j$. Let $c=\min _{i=1, \ldots, N} \phi\left(e_{i}\right)>0, f=$ $\min _{i=1, \ldots, N} f_{e_{i}}$, and choose $\alpha, \beta>0$ such that $\alpha t \wedge \beta \leq f(t)$ for all $t \geq 0$. We have:

$$
F_{h}\left(u_{h}\right) \geq 2 c h^{N} \sum_{x \in h \mathbb{Z}^{N}} \sum_{i=1}^{N} \alpha\left|\frac{u_{h}(x)-u_{h}\left(x+h e_{i}\right)}{h}\right|^{2} \wedge \frac{\beta}{h}
$$

(remember we only sum on $x$ such that $x, x+h e_{i} \in \Omega$ ). We first show that the sequence $\left(u_{h}\right)$ is bounded in $B V_{l o c}(\Omega)$ (so that it is compact in $L_{l o c}^{1}(\Omega)$ ). Choose $R>0, i \in\{1, \ldots, N\}$, and write (with $\Omega_{\frac{1}{2} \sqrt{N} h}=$ $\left.\left\{x \in \Omega: \operatorname{dist}(x, \partial \Omega)>\frac{1}{2} \sqrt{N} h\right\}\right)$

$$
\begin{aligned}
\left|D_{i} u_{h}\right|\left(\Omega_{\frac{1}{2} \sqrt{N} h} \cap B_{R}(0)\right) & \leq \sum_{x \in h \mathbb{Z}^{N} \cap B_{R}(0)} h^{N-1}\left|u_{h}(x)-u_{h}\left(x+h e_{i}\right)\right| \\
& \leq \sum_{x \in X_{+}} 2\left\|u_{h}\right\|_{\infty} h^{N-1}+h^{N} \sum_{x \in X_{-}}\left|\frac{u_{h}(x)-u_{h}\left(x+h e_{i}\right)}{h}\right|
\end{aligned}
$$

where $X_{+}=\left\{x \in h \mathbb{Z}^{N} \cap B_{R}(0):\left|u_{h}(x)-u_{h}\left(x+h e_{i}\right)\right|>\sqrt{\beta h / \alpha}\right\}$ and $X_{-}=h \mathbb{Z}^{N} \cap B_{R}(0) \backslash X_{+}$. Of course, we only consider points $x \in h \mathbb{Z}^{N}$ such that $x$ and $x+h e_{i}$ belong to $\Omega$. Then, using the Cauchy-Schwarz inequality,

$$
\begin{aligned}
\left|D_{i} u_{h}\right|\left(\Omega_{\frac{1}{2} \sqrt{N} h} \cap B_{R}(0)\right) & \leq 2\left\|u_{h}\right\|_{\infty} h^{N-1} \operatorname{card} X_{+}+C R^{\frac{N}{2}}\left\{h^{N} \sum_{x \in X_{-}}\left|\frac{u_{h}(x)-u_{h}\left(x+h e_{i}\right)}{h}\right|^{2}\right\}^{\frac{1}{2}} \\
& \leq \frac{\left\|u_{h}\right\|_{\infty} F_{h}\left(u_{h}\right)}{\beta c}+C R^{\frac{N}{2}} \sqrt{\frac{F_{h}\left(u_{h}\right)}{2 \alpha c}}
\end{aligned}
$$

with $C$ some constant depending only on $N$, so that eventually, for any $\eta>0$,

$$
\sup _{h}\left|D u_{h}\right|\left(\Omega_{\eta} \cap B_{R}(0)\right)<+\infty .
$$


This shows that upon extracting a subsequence we may assume that $u_{h}$ converges almost everywhere in $\Omega$ (and in $L_{l o c}^{p}(\Omega)$, as well) to some function $u$ that belongs to $B V\left(\Omega \cap B_{R}(0)\right)$ for any $R>0$.

Now consider the extension of $u_{h}$ (on $\mathbb{R}^{N}, u_{h}(x)$ being considered to be 0 outside of $\Omega$ )

$$
v_{h}(y)=\sum_{x \in h \mathbb{Z}^{N}} u_{h}(x) \Delta_{N}\left(\frac{y-x}{h}\right)
$$

where $\Delta(t)=(1-|t|)^{+}$for any $t \in \mathbb{R}$ and $\Delta_{N}(y)=\prod_{i=1}^{N} \Delta\left(y_{i}\right)$ for any $y \in \mathbb{R}^{N}$. We estimate $\int\left|\nabla v_{h}\right|^{2}$ on an "elementary cell", for instance $(0, h)^{N}$ :

$$
\begin{aligned}
\int_{(0, h)^{N}}\left|\partial_{1} v_{h}(y)\right|^{2} d y= & \int_{(0, h)^{N}}\left|\sum_{x \in\{0, h\}^{N}} u_{h}(x) \frac{1}{h} \Delta^{\prime}\left(\frac{y_{1}-x_{1}}{h}\right) \prod_{i=2}^{N} \Delta\left(\frac{y_{i}-x_{i}}{h}\right)\right|^{2} d y \\
= & h \int_{(0, h)^{N-1}} d y_{2} \ldots d y_{N}\left|\sum_{x \in\{0, h\}^{N-1}} \frac{u_{h}(h, x)-u_{h}(0, x)}{h} \prod_{i=2}^{N} \Delta\left(\frac{y_{i}-x_{i}}{h}\right)\right|^{2} \\
= & h \int_{(0, h)^{N-2}} d y_{3} \ldots d y_{N} \int_{0}^{h} \mid\left(\frac{y_{2}}{h}\right) \sum_{x \in\{0, h\}^{N-2}} \frac{u_{h}(h, h, x)-u_{h}(0, h, x)}{h} \prod_{i=3}^{N} \Delta\left(\frac{y_{i}-x_{i}}{h}\right) \\
& +\left(1-\frac{y_{2}}{h}\right) \sum_{x \in\{0, h\}^{N-2}} \frac{u_{h}(h, 0, x)-u_{h}(0,0, x)}{h} \prod_{i=3}^{N} \Delta\left(\frac{y_{i}-x_{i}}{h}\right)^{2} d y_{2} \\
\leq & \frac{h^{2}}{2}\left\{\int_{(0, h)^{N-2}} d y_{3} \ldots d y_{N}\left|\sum_{x \in\{0, h\}^{N-2}} \frac{u_{h}(h, h, x)-u_{h}(0, h, x)}{h} \prod_{i=3}^{N} \Delta\left(\frac{y_{i}-x_{i}}{h}\right)\right|^{2}\right. \\
& \left.+\int_{(0, h)^{N-2}} d y_{3} \ldots d y_{N}\left|\sum_{x \in\{0, h\}^{N-2}} \frac{u_{h}(h, 0, x)-u_{h}(0,0, x)}{h} \prod_{i=3}^{N} \Delta\left(\frac{y_{i}-x_{i}}{h}\right)\right|^{2}\right\}
\end{aligned}
$$

by induction we deduce that

$$
\int_{(0, h)^{N}}\left|\partial_{1} v_{h}(y)\right|^{2} d y \leq \frac{h^{N}}{2^{N-1}} \sum_{x \in\{0, h\}^{N-1}}\left|\frac{u_{h}(h, x)-u_{h}(0, x)}{h}\right|^{2} .
$$

Notice that we could therefore conclude that

$$
\int_{\Omega_{\sqrt{N} h}}\left|\nabla v_{h}(y)\right|^{2} d y \leq h^{N} \sum_{x \in h \mathbb{Z}^{N}} \sum_{i=1}^{N}\left|\frac{u_{h}(x)-u_{h}\left(x+h e_{i}\right)}{h}\right|^{2}
$$

(with $\Omega_{\sqrt{N} h}=\{x \in \Omega: \operatorname{dist}(x, \partial \Omega)>\sqrt{N} h\}$, since we control the gradient of $v_{h}$ only on the cubes $x+(0, h)^{N}$, $x \in h \mathbb{Z}^{N}$ whose $2^{N}$ vertices all belong to $\Omega$ ), but since we can not control the right-hand side of this expression 
if it is summed over all $x$ we must introduce a slight modification of $v_{h}$ : we define thus $\hat{v}_{h}=v_{h}$, except each time

$$
\left|u_{h}(x)-u_{h}\left(x+h e_{i}\right)\right|>\sqrt{\frac{\beta h}{\alpha}}
$$

in which case we set $\hat{v}_{h} \equiv 0$ on $\left(x, x+h e_{i}\right) \times \prod_{i^{\prime} \neq i}\left(x-h e_{i^{\prime}}, x+h e_{i^{\prime}}\right)=U_{x, e_{i}}^{h}$. The new function $\hat{v}_{h}$ is in $S B V_{l o c}(\Omega)$, and $S_{\hat{v}_{h}} \subseteq \bigcup_{\left(x, e_{i}\right) \in X_{h}} \partial U_{x, e_{i}}^{h}$ where the union is taken on $X_{h}=\left\{\left(x, e_{i}\right):\right.$ (5) holds $\}$. Now, we can write

$$
\int_{\Omega_{\sqrt{N} h}}\left|\nabla \hat{v}_{h}(y)\right|^{2} d y \leq \sum_{\left(x, e_{i}\right) \in h \mathbb{Z}^{N} \backslash X_{h}}\left|\frac{u_{h}(x)-u_{h}\left(x+h e_{i}\right)}{h}\right|^{2} \leq \frac{F_{h}\left(u_{h}\right)}{2 c \alpha},
$$

moreover since $\mathcal{H}^{N-1}\left(\partial U_{x, e_{i}}^{h}\right)=\kappa h^{N-1}\left(\right.$ with $\left.\kappa=2^{N-1}(N+1)\right)$,

$$
\mathcal{H}^{N-1}\left(\Omega_{\sqrt{N} h} \cap S_{\hat{v}_{h}}\right) \leq \operatorname{card} X_{h} \kappa h^{N-1} \leq \kappa \frac{F_{h}\left(u_{h}\right)}{2 c \beta} .
$$

From (6) and (7) and $\operatorname{since} \sup _{h}\left\|\hat{v}_{h}\right\|_{\infty}<+\infty$, we deduce invoking Ambrosio's Theorem 3 (see Appendix A) that some subsequence of $\hat{v}_{h}$ converges to a function $v \in L^{\infty}(\Omega) \cap S B V_{l o c}(\Omega)$, with

$$
\int_{\Omega}|\nabla v(x)|^{2} d x+\mathcal{H}^{N-1}\left(S_{v}\right)=\sup _{A \subset \subset \Omega} \int_{A}|\nabla v(x)|^{2} d x+\mathcal{H}^{N-1}\left(A \cap S_{v}\right) \leq \frac{1}{2 c}\left(\frac{1}{\alpha}+\frac{\kappa}{\beta}\right) \liminf _{h \downarrow 0} F_{h}\left(u_{h}\right)<+\infty .
$$

The proof of the lemma is achieved once we notice that $v$ must be equal to $u$ (as for instance by the construction of $v_{h}$ and $\hat{v}_{h}$ it is simple to check that for any $A \subset \subset \Omega$ with regular boundary $\int_{A}\left(u_{h}(y)-\hat{v}_{h}(y)\right) d y \rightarrow 0$ as $h \downarrow 0)$.

Remark. If we drop the condition $\phi\left(e_{i}\right)>0$ for $i=1, \ldots, N$, the result may be false. For instance, if $N=1$, $\phi \equiv 0$ except at -2 and 2 where $\phi(-2)=\phi(2)=1$, the family $\left(u_{h}\right)_{h>0}$ defined by

$$
u_{h}(k h)=\left\{\begin{array}{ll}
0 & \text { if } k \in 2 \mathbb{Z} \\
1 & \text { if } k \in 2 \mathbb{Z}+1
\end{array} \quad \text { for every } k \in \mathbb{Z}\right.
$$

satisfies the assumptions of Lemma 1 but is not compact.

\subsection{Estimate from below of the $\Gamma$-limit}

In this section we wish to prove that for all $u \in L^{p}(\Omega)$,

$$
F(u) \leq F^{\prime}(u) .
$$

We must therefore prove that for any $u \in L^{p}(\Omega)$ and any sequence $\left(u_{h_{j}}\right)$ that converges to $u$ in $L^{p}(\Omega)$ as $j \rightarrow \infty$ (with $\lim _{j \rightarrow \infty} h_{j}=0$ ) we have,

$$
F(u) \leq \liminf _{j \rightarrow \infty} F_{h_{j}}\left(u_{h_{j}}\right) .
$$

Let $u \in L^{p}(\Omega)$, and we will suppose first that it is bounded. Choose also an arbitrary decreasing sequence $h_{j} \downarrow 0$ and functions $u_{h_{j}}$ that converge to $u$ in $L^{p}(\Omega)$. We can assume that $\left\|u_{h_{j}}\right\|_{\infty} \leq\|u\|_{\infty}$, as truncating $u_{h_{j}}$ we decrease its energy $F_{h_{j}}\left(u_{h_{j}}\right)$. It is clearly not restrictive to consider, as well, that the lim inf is in fact a limit, 
and that $\sup _{j} F_{h_{j}}\left(u_{h_{j}}\right)<+\infty$ (since if $\liminf _{j \rightarrow \infty} F_{h_{j}}\left(u_{h_{j}}\right)=+\infty$ the result is obvious). In view of Lemma 1 we deduce that

$$
u \in S B V_{l o c}(\Omega) \quad \text { and } \quad \int_{\Omega}|\nabla u(x)|^{2} d x+\mathcal{H}^{N-1}\left(S_{u}\right)<+\infty .
$$

In the sequel we will drop the subscripts $j$ and write " $h \downarrow 0$ " for " $j \rightarrow \infty$ ". We prove (9) following Gobbino's method in [21], with a few modifications and adaptations. Let

$$
\hat{\Omega}_{h}=\bigcup_{x \in h \mathbb{Z}^{N} \cap \Omega} x+\left[-\frac{h}{2}, \frac{h}{2}\right]^{N}
$$

and notice that $\Omega_{\frac{1}{2} \sqrt{N} h}=\left\{x \in \Omega: \operatorname{dist}(x, \partial \Omega)>\frac{1}{2} \sqrt{N} h\right\} \subset \hat{\Omega}_{h}$. We have (still using the convention that we only consider in the sums the points that fall inside $\Omega$ )

$$
\begin{aligned}
F_{h}\left(u_{h}\right) & =h^{N} \sum_{x \in h \mathbb{Z}^{N}} \sum_{\xi \in \mathbb{Z}^{N}} \frac{1}{h} f_{\xi}\left(\frac{\left(u_{h}(x)-u_{h}(x+h \xi)\right)^{2}}{h}\right) \phi(\xi) \\
& =\int_{\hat{\Omega}_{h}} d y \sum_{\xi \in \mathbb{Z}^{N} \cap \frac{1}{h}\left(\hat{\Omega}_{h}-y\right)} \frac{1}{h} f_{\xi}\left(\frac{\left(u_{h}(y)-u_{h}(y+h \xi)\right)^{2}}{h}\right) \phi(\xi) \\
& =\sum_{\xi \in \mathbb{Z}^{N}} \phi(\xi) \int_{\hat{\Omega}_{h} \cap\left(\hat{\Omega}_{h}-h \xi\right)} \frac{1}{h} f_{\xi}\left(\frac{\left(u_{h}(y)-u_{h}(y+h \xi)\right)^{2}}{h}\right) d y
\end{aligned}
$$

For every $\xi \in \mathbb{Z}^{N}$ we let

$$
\hat{F}_{h}\left(u_{h}, \xi\right)=\int_{\hat{\Omega}_{h} \cap\left(\hat{\Omega}_{h}-h \xi\right)} \frac{1}{h} f_{\xi}\left(\frac{\left(u_{h}(y)-u_{h}(y+h \xi)\right)^{2}}{h}\right) d y .
$$

Inequality (9) will follow by Fatou's lemma if we prove that for any $\xi$,

$$
\liminf _{h \downarrow 0} \hat{F}_{h}\left(u_{h}, \xi\right) \geq \alpha_{\xi} \int_{\Omega}|\langle\nabla u(x), \xi\rangle|^{2} d x+\beta_{\xi} \int_{S_{u}}\left|\left\langle\nu_{u}(x), \xi\right\rangle\right| d \mathcal{H}^{N-1}(x) .
$$

We choose $A \subset \subset \Omega$. If $h$ is small enough (i.e., $\left.h \leq \operatorname{dist}(A, \partial \Omega) /\left(|\xi|+\frac{1}{2} \sqrt{N}\right)\right)$ then

$$
\hat{F}_{h}\left(u_{h}, \xi\right) \geq \int_{A} \frac{1}{h} f_{\xi}\left(\frac{\left(u_{h}(y)-u_{h}(y+h \xi)\right)^{2}}{h}\right) d y
$$

and it will be sufficient to show that

$$
\liminf _{h \downarrow 0} \int_{A} \frac{1}{h} f_{\xi}\left(\frac{\left(u_{h}(y)-u_{h}(y+h \xi)\right)^{2}}{h}\right) d y \geq \alpha_{\xi} \int_{A}|\langle\nabla u(x), \xi\rangle|^{2} d x+\beta_{\xi} \int_{S_{u} \cap A}\left|\left\langle\nu_{u}(x), \xi\right\rangle\right| d \mathcal{H}^{N-1}(x),
$$

as the supremum of the right-hand side of (12) for all $A \subset \subset \Omega$ is the right-hand side of (11). This is part of Gobbino's result [21], but we present a slightly different approach, still based on the "slicing" (see Appendix A for technical details) of the functions $u_{h}$ in the direction $\xi$. 
Let $\xi^{\perp}=\left\{z \in \mathbb{R}^{N}:\langle z, \xi\rangle=0\right\}$, and for every $z \in \xi^{\perp}, A_{z, \xi}=\{s \in \mathbb{R}: z+s \xi \in A\},\left(u_{h}\right)_{z, \xi}(s)=u_{h}(z+s \xi)$. We rewrite the first integral over $A$ in (12):

$$
\begin{aligned}
\int_{\xi^{\perp}} d \mathcal{H}^{N-1}(z) & \int_{A_{z, \xi}} \frac{1}{h} f_{\xi}\left(\frac{\left(\left(u_{h}\right)_{z, \xi}(s)-\left(u_{h}\right)_{z, \xi}(s+h)\right)^{2}}{h}\right)|\xi| d s= \\
= & |\xi| \int_{\xi^{\perp}} d \mathcal{H}^{N-1}(z) \sum_{k \in \mathbb{Z}} \int_{A_{z, \xi} \cap[k h, k h+h)} \frac{1}{h} f_{\xi}\left(\frac{\left(\left(u_{h}\right)_{z, \xi}(s)-\left(u_{h}\right)_{z, \xi}(s+h)\right)^{2}}{h}\right) d s \\
= & |\xi| \int_{\xi^{\perp}} d \mathcal{H}^{N-1}(z) \int_{[0, h)} d t\left\{\sum_{k \in \mathbb{Z}} \frac{1}{h} f_{\xi}\left(\frac{\left(\left(u_{h}\right)_{z, \xi}(t+k h)-\left(u_{h}\right)_{z, \xi}(t+(k+1) h)\right)^{2}}{h}\right)\right\}
\end{aligned}
$$

(by the change of variable $t+k h=s$ ) where the sum is taken only on the $k \in \mathbb{Z}$ such that $t+k h \in A_{z, \xi}$. Now, with the change of variable $t=h \tau$, this becomes

$$
|\xi| \int_{\xi^{\perp}} d \mathcal{H}^{N-1}(z) \int_{0}^{1} d \tau\left\{h \sum_{k \in \mathbb{Z}} \frac{1}{h} f_{\xi}\left(\frac{\left(\left(u_{h}\right)_{z, \xi}((\tau+k) h)-\left(u_{h}\right)_{z, \xi}((\tau+k+1) h)\right)^{2}}{h}\right)\right\}
$$

We will prove that for a.e. $(z, \tau) \in \xi^{\perp} \times(0,1)$,

$$
\begin{aligned}
\liminf _{h \downarrow 0} h \sum_{\left\{\begin{array}{l}
k \in \mathbb{Z} \\
(\tau+k) h \in A_{z, \xi}
\end{array}\right.} \frac{1}{h} f_{\xi}\left(\frac{\left(\left(u_{h}\right)_{z, \xi}((\tau+k) h)-\left(u_{h}\right)_{z, \xi}((\tau+k+1) h)\right)^{2}}{h}\right) \geq \\
\quad \geq \alpha_{\xi} \int_{A_{z, \xi}}\left|u_{z, \xi}^{\prime}(x)\right|^{2} d x+\beta_{\xi} \mathcal{H}^{0}\left(S_{u_{z, \xi}} \cap A_{z, \xi}\right) .
\end{aligned}
$$

In order to prove (13), we need some information on the limit of $\left(\left(u_{h}\right)_{z, \xi}((\tau+k) h)\right)_{k \in \mathbb{Z}}$ as $h \downarrow 0$. Since, using the same changes of variables,

$$
\begin{aligned}
\int_{\Omega}\left|u_{h}(y)-u(y)\right|^{p} d y & =\int_{\xi^{\perp}} d \mathcal{H}^{N-1}(z) \int_{\Omega_{z, \xi}}\left|\left(u_{h}\right)_{z, \xi}(s)-u_{z, \xi}(s)\right|^{p}|\xi| d s \\
& =|\xi| \int_{\xi^{\perp}} d \mathcal{H}^{N-1}(z) \int_{0}^{1} d \tau\left\{h \sum_{k \in \mathbb{Z}}\left|\left(u_{h}\right)_{z, \xi}((\tau+k) h)-u_{z, \xi}((\tau+k) h)\right|^{p}\right\}
\end{aligned}
$$

(where in the sum we consider only $k$ such that $\left.(\tau+k) h \in \Omega_{z, \xi}\right)$ we may assume (upon extracting a subsequence) that for a.e. $(z, \tau) \in \xi^{\perp} \times(0,1)$,

$$
\lim _{h \downarrow 0} h \sum_{k \in \mathbb{Z}}\left|\left(u_{h}\right)_{z, \xi}((\tau+k) h)-u_{z, \xi}((\tau+k) h)\right|^{p}=0 .
$$

Choose a $(z, \tau)$ such that (14) holds. By (10) we may also assume when choosing $z$ that

$$
u_{z, \xi} \in S B V_{l o c}\left(\Omega_{z, \xi}\right) \quad \text { and } \quad \int_{\Omega_{z, \xi}}\left|u_{z, \xi}^{\prime}(s)\right|^{2} d s+\mathcal{H}^{0}\left(S_{u_{z, \xi}}\right)<+\infty,
$$

so that $u_{z, \xi}$ is continuous except at a finite number of points. Thus, for almost all $s \in \Omega_{z, \xi}$,

$$
\lim _{h \downarrow 0} u_{z, \xi}\left(\left(\tau+\left[\frac{s}{h}\right]\right) h\right)=u_{z, \xi}(s)
$$


(where [.] denotes the integer part). We easily deduce from this and (14) that the piecewise constant function $v_{h}: \Omega_{z, \xi} \rightarrow \mathbb{R}$ defined by

$$
v_{h}(s)=\left(u_{h}\right)_{z, \xi}\left(\left(\tau+\left[\frac{s}{h}\right]\right) h\right)
$$

converges to $u_{z, \xi}$ in $L_{l o c}^{p}\left(\Omega_{z, \xi}\right)$.

Remark. Following Gobbino (proof of Lemma 3.3, Step 2 in [21]) we could also prove that for a.e. $\tau \in(0,1)$, $u_{z, \xi}((\tau+[s / h]) h) \rightarrow u_{z, \xi}(s)$ in $L_{l o c}^{1}\left(\Omega_{z, \xi}\right)$, so that the a priori information on the regularity of $u$ is not really needed.

We return to the proof of inequality (13). For any $I \subset \subset A_{z, \xi}$, we denote

$$
\begin{aligned}
G\left(v_{h}, I\right) & =\int_{I} \frac{1}{h} f_{\xi}\left(\frac{\left|v_{h}(s+h)-v_{h}(s)\right|^{2}}{h}\right) d s \\
& =\sum_{k \in \mathbb{Z}}|(k h, k h+h) \cap I| \frac{1}{h} f_{\xi}\left(\frac{\left|v_{h}((k+1) h)-v_{h}(k h)\right|^{2}}{h}\right) .
\end{aligned}
$$

If $h$ is small enough, $(\tau+[s / h]) h \in A_{z, \xi}$ for every $s \in I$ so that the lim inf in (13) is greater than $\liminf _{h \downarrow 0} G\left(v_{h}, I\right)$. Therefore, we just need to prove that for any $I \subset \subset A_{z, \xi}$,

$$
\liminf _{h \downarrow 0} G\left(v_{h}, I\right) \geq \alpha_{\xi} \int_{I}\left|u_{z, \xi}^{\prime}(s)\right|^{2} d s+\beta_{\xi} \mathcal{H}^{0}\left(S_{u_{z, \xi}} \cap I\right) ;
$$

indeed, taking then the lowest greater bound of the right-hand term of (15) for all $I$, we will get (13). Because of the super-additivity of $\liminf _{h \downarrow 0} G\left(v_{h}, \cdot\right)$ we may assume without loss of generality that $I$ is an interval. To prove (15), we then choose $\alpha, \beta>0$ such that $\alpha t \wedge \beta \leq f_{\xi}(t)$ for all $t \geq 0$ (noticing that $\alpha$-respectively, $\beta$-may be chosen as close as wanted to $\alpha_{\xi}-$ resp., $\beta_{\xi}$ ), and we write

$$
G\left(v_{h}, I\right) \geq \sum_{(k h, k h+h) \subset I} h \alpha\left|\frac{v_{h}((k+1) h)-v_{h}(k h)}{h}\right|^{2} \wedge \beta .
$$

Redefining a function $\tilde{v}_{h}$ with $\tilde{v}_{h}(k h)=v_{h}(k h)$ for $k h \in I$, affine on the intervals $(k h, k h+h) \subset I$ such that $h \alpha\left|\frac{v_{h}((k+1) h)-v_{h}(k h)}{h}\right|^{2} \leq \beta$ and piecewise constant, jumping once on the intervals with the reverse inequality (just like in [9]), we get

$$
G\left(v_{h}, I\right) \geq \alpha \int_{I_{h}}\left|\tilde{v}_{h}^{\prime}(s)\right|^{2} d s+\beta \mathcal{H}^{0}\left(S_{\tilde{v}_{h}} \cap I_{h}\right)
$$

with $I_{h}=\{x \in I: \operatorname{dist}(x, \mathbb{R} \backslash I)>h\}$, so that invoking Theorem 3 (Appendix A) we get the existence of a function $\tilde{v}$ such that some subsequence of $\tilde{v}_{h}$ goes to $\tilde{v}$ a.e., and that satisfies

$$
\alpha \int_{I}\left|\tilde{v}^{\prime}(s)\right|^{2} d s+\beta \mathcal{H}^{0}\left(S_{\tilde{v}} \cap I\right) \leq \liminf _{h \downarrow 0} G\left(v_{h}, I\right) .
$$

We check then that $\tilde{v}$ has to be equal to $u_{z, \xi}$ (noticing easily, for instance, that $\left(v_{h}-\tilde{v}_{h}\right) \rightarrow 0$ weakly in $L^{p}$ ). If $\alpha \rightarrow \alpha_{\xi}$ we deduce from (16)

$$
\alpha_{\xi} \int_{I}\left|u_{z, \xi}^{\prime}(s)\right|^{2} d s \leq \liminf _{h \downarrow 0} G\left(v_{h}, I\right)
$$


whereas sending $\beta$ to $\beta_{\xi}$ we get

$$
\beta_{\xi} \mathcal{H}^{0}\left(S_{u_{z, \xi}} \cap I\right) \leq \liminf _{h \downarrow 0} G\left(v_{h}, I\right) .
$$

Inequality (15) is deduced from the last two inequalities by subdividing the interval $I$ into suitable subintervals (the connected components of a small neighborhood of $S_{u_{z, \xi}}$ and its complement) and using the appropriate inequality in each subinterval. Hence (13) holds, and using Fatou's lemma we deduce (12), as

$$
\begin{aligned}
|\xi| \int_{\xi^{\perp}} d \mathcal{H}^{N-1}(z)\left(\alpha_{\xi} \int_{A_{z, \xi}}\left|u_{z, \xi}^{\prime}(s)\right|^{2}+\beta_{\xi} \mathcal{H}^{0}\left(S_{u_{z, \xi}} \cap A_{z, \xi}\right)\right)= \\
\quad=\alpha_{\xi} \int_{A}|\langle\nabla u(x), \xi\rangle|^{2} d x+\beta_{\xi} \int_{S_{u} \cap A}\left|\left\langle\nu_{u}(x), \xi\right\rangle\right| d \mathcal{H}^{N-1}(x) .
\end{aligned}
$$

Inequality (9) therefore holds in the case $u \in L^{\infty}(\Omega)$.

Now, if $u \in L^{p}(\Omega)$ is not bounded, choose again $u_{h_{j}} \rightarrow u$ in $L^{p}(\Omega)$. Consider $u^{k}=(-k \vee u) \wedge k$ and $u_{h_{j}}^{k}=\left(-k \vee u_{h_{j}}\right) \wedge k$, clearly $u_{h_{j}}^{k} \rightarrow u^{k}$ in $L^{p}(\Omega)$, so that

$$
F\left(u^{k}\right) \leq \liminf _{j \rightarrow \infty} F_{h_{j}}\left(u_{h_{j}}^{k}\right)
$$

But as $f$ is increasing, $F_{h_{j}}\left(u_{h_{j}}^{k}\right) \leq F_{h_{j}}\left(u_{h_{j}}\right)$ so that

$$
F\left(u^{k}\right) \leq \liminf _{j \rightarrow \infty} F_{h_{j}}\left(u_{h_{j}}\right) .
$$

If this is finite, we conclude by noticing that $\lim _{k \rightarrow \infty} F\left(u^{k}\right)=F(u)$ (by (21), (22)); so that the proof of (8) is achieved.

Remark. Notice that if $u_{h_{j}} \rightarrow u$ in $L_{l o c}^{p}(\Omega)$, the result still holds. Indeed, for any $A \subset \subset \Omega$ we have $u_{h_{j}} \rightarrow u$ in $L^{p}(A)$ and since the result holds in this case we can write

$$
F(u, A) \leq \liminf _{j \rightarrow \infty} F_{h_{j}}\left(u_{h_{j}}, A\right) \leq \liminf _{j \rightarrow \infty} F_{h_{j}}\left(u_{h_{j}}, \Omega\right) .
$$

Then, as $F(u, \Omega)=\sup _{A \subset \subset \Omega} F(u, A)$ we get (9). (Thus the $F_{h}$ also $\Gamma$-converge to $F$ in $L^{p}(\Omega)$ endowed with the $L_{l o c}^{p}(\Omega)$ topology.)

\subsection{Estimate from above of the $\Gamma$-limit}

Given $u \in G S B V_{l o c}(\Omega) \cap L^{p}(\Omega)$ with $F(u)=F(u, \Omega)<+\infty$, we want to build $u_{h} \in \ell^{p}\left(\Omega \cap h \mathbb{Z}^{N}\right)$ such that $u_{h} \rightarrow u$ in $L^{p}(\Omega)$ and

$$
\limsup _{h \downarrow 0} F_{h}\left(u_{h}\right) \leq F(u) .
$$

In order to be able to assume some regularity on the function $u$ we first prove the following lemma.

Lemma 2. Let $u \in G S B V_{l o c}(\Omega) \cap L^{p}(\Omega)$ with $F(u)<+\infty$. There exists a sequence $\left(u_{k}\right)_{k \geq 1} \subseteq S B V(\Omega)$ of bounded functions with bounded supports, that are almost everywhere continuous in $\Omega$ and such that

- $u_{k} \rightarrow u$ in $L^{p}(\Omega)$ as $k$ goes to infinity,

- $\lim _{k \rightarrow \infty} F\left(u_{k}\right)=F(u)$. 
Remark. The information on the support of $u_{k}$ makes sense only when $\Omega$ is unbounded.

Proof. For every integer $k \geq 1$ let first $u^{k}=(-k \vee u) \wedge k$ be the truncated of $u$ at level $k$. We choose in $L^{p}\left(\mathbb{R}^{N}\right)$ a minimizer $v_{k}$ of

$$
v \mapsto F(v)+k \int_{\mathbb{R}^{N}}\left|v(x)-u^{k}(x)\right|^{p} d x
$$

Then,

$$
\begin{aligned}
& \left\|v_{k}-u\right\|_{L^{p}\left(\mathbb{R}^{N}\right)} \leq\left\|v_{k}-u^{k}\right\|_{L^{p}\left(\mathbb{R}^{N}\right)}+\left\|u^{k}-u\right\|_{L^{p}\left(\mathbb{R}^{N}\right)} \leq \\
& \leq\left(\frac{1}{k} F\left(u^{k}\right)\right)^{\frac{1}{p}}+\left(\int_{\{|u|>k\}}(|u(x)|-k)^{p} d x\right)^{\frac{1}{p}} \leq\left(\frac{1}{k} F(u)\right)^{\frac{1}{p}}+\left(\int_{\{|u|>k\}}|u(x)|^{p} d x\right)^{\frac{1}{p}} \rightarrow 0
\end{aligned}
$$

as $k \rightarrow \infty$, moreover by Lemma 3 (see Appendix A.2) we know that $\mathcal{H}^{N-1}\left(\Omega \cap \overline{S_{v_{k}}} \backslash S_{u}\right)=0$ and $v_{k} \in C^{1}\left(\Omega \backslash \overline{S_{v_{k}}}\right)$ so that in particular $v_{k}$ is almost everywhere continuous. We also have that $F\left(v_{k}\right) \leq F\left(u^{k}\right) \leq F(u)$ and $\left|v_{k}(x)\right| \leq k$ for all $x \in \Omega$. Set now for every integer $n>1$ and $x \in \Omega$

$$
v_{k, n}(x)= \begin{cases}v_{k}(x)-\frac{1}{n} & \text { if } v_{k}(x)>1 / n \\ 0 & \text { if }\left|v_{k}(x)\right| \leq 1 / n ; \text { and } \\ v_{k}(x)+\frac{1}{n} & \text { if } v_{k}(x)<-1 / n\end{cases}
$$

Clearly $v_{k, n}$ is still a.e. continuous and goes to $v_{k}$ in $L^{p}(\Omega)$ as $n \rightarrow \infty$, so that we can choose $n_{k}$ such that $\left\|v_{k, n_{k}}-v_{k}\right\|_{L^{p}(\Omega)} \leq 1 / k$. We set $w_{k}=v_{k, n_{k}}$.

We also have $S_{w_{k}} \subseteq S_{v_{k}}$,

$$
\nabla w_{k}= \begin{cases}\nabla v_{k} & \text { a.e. in }\left\{x \in \Omega:\left|v_{k}(x)\right|>1 / n_{k}\right\} \\ 0 & \text { a.e. in the complement }\end{cases}
$$

and

$$
\left|\left\{w_{k} \neq 0\right\}\right|=\left|\left\{\left|v_{k}\right|>\frac{1}{n_{k}}\right\}\right| \leq n_{k}^{p} \int_{\Omega}\left|v_{k}(x)\right|^{p} d x<+\infty
$$

so that in particular $w_{k} \in L^{q}(\Omega)$ for any $q \in[1,+\infty]$.

Choose at last $\zeta \in C_{0}^{\infty}\left(\mathbb{R}^{N}\right)$ with $0 \leq \zeta \leq 1$ and $\zeta \equiv 1$ on $B_{1}(0)$, and set for $R>0$ and any $x \in \Omega$ $w_{k, R}(x)=\zeta\left(\frac{x}{R}\right) w_{k}(x)$. For any $R$,

$$
S_{w_{k, R}} \subseteq S_{w_{k}} \subseteq S_{v_{k}}
$$

and if $\xi \in \mathbb{Z}^{N}$,

$$
\begin{aligned}
\int_{\Omega}\left|\left\langle\nabla w_{k, R}(x), \xi\right\rangle\right|^{2} d x= \\
\quad=\int_{B_{R}(0) \cap \Omega}\left|\left\langle\nabla w_{k}(x), \xi\right\rangle\right|^{2} d x+\int_{\Omega \backslash B_{R}(0)}\left|\zeta\left(\frac{x}{R}\right)\left\langle\nabla w_{k}(x), \xi\right\rangle+\frac{w_{k}(x)}{R}\left\langle\nabla \zeta\left(\frac{x}{R}\right), \xi\right\rangle\right|^{2} d x \\
\leq \int_{B_{R}(0) \cap \Omega}\left|\left\langle\nabla v_{k}(x), \xi\right\rangle\right|^{2} d x+2 \int_{\Omega \backslash B_{R}(0)}\left|\left\langle\nabla v_{k}(x), \xi\right\rangle\right|^{2} d x+\frac{C}{R^{2}}|\xi|^{2} \int_{\Omega \backslash B_{R}(0)}\left|w_{k}(x)\right|^{2} d x
\end{aligned}
$$


with $C=2\|\nabla \zeta\|_{L^{\infty}\left(\mathbb{R}^{N}\right)}^{2}$. Hence

$$
F\left(w_{k, R}\right) \leq F\left(v_{k}\right)+\left(\sum_{\xi \in \mathbb{Z}^{N}} \alpha_{\xi} \phi(\xi)|\xi|^{2}\right)\left\{\int_{\Omega \backslash B_{R}(0)}\left|\nabla v_{k}(x)\right|^{2} d x+\frac{C}{R^{2}} \int_{\Omega \backslash B_{R}(0)}\left|w_{k}(x)\right|^{2} d x\right\} .
$$

Since $w_{k}$ and $\nabla v_{k}$ are in $L^{2}(\Omega)$, we can choose $R$ large enough in order to have

$$
F\left(w_{k, R}\right) \leq F\left(v_{k}\right)+\frac{1}{k}
$$

Choose $R_{k}$ large enough so that (18) holds and $\left\|w_{k, R_{k}}-w_{k}\right\|_{L^{p}(\Omega)} \leq 1 / k$, and set $u_{k}=w_{k, R_{k}}$. Clearly $u_{k}$ is still a.e. continuous. Moreover, $F\left(u_{k}\right) \leq F(u)+1 / k, u_{k}$ goes to $u$ in $L^{p}(\Omega)$ as $k \rightarrow \infty$, and by Theorem 3 (Appendix A) we deduce that

$$
F(u) \leq \liminf _{k \rightarrow \infty} F\left(u_{k}\right)
$$

so that $\lim _{k \rightarrow \infty} F\left(u_{k}\right)=F(u)$ and the lemma is true.

We now establish (17). First consider the case $\Omega=\mathbb{R}^{N}$. Given $u \in G S B V_{\text {loc }}\left(\mathbb{R}^{N}\right) \cap L^{p}\left(\mathbb{R}^{N}\right)$ with $F(u)<+\infty$, we build invoking Lemma 2 a sequence of compactly supported, bounded and a.e. continuous functions $u_{k}$ converging to $u$ such that $F\left(u_{k}\right) \rightarrow F(u)$ as $k$ goes to infinity. By a standard diagonalization procedure, if we know how to build for every $k$ a sequence $\left(\left(u_{k}\right)_{h}\right)_{h>0}$ converging to $u_{k}$ in $L^{p}\left(\mathbb{R}^{N}\right)$ as $h \downarrow 0$, such that

$$
\limsup _{h \downarrow 0} F_{h}\left(\left(u_{k}\right)_{h}\right) \leq F\left(u_{k}\right),
$$

we will be able to find $u_{h}$ with $u_{h} \rightarrow u$ and satisfying (17). In the sequel we may therefore assume that $u$ is bounded, compactly supported, and continuous at almost every $x \in \mathbb{R}^{N}$.

For $y \in(0, h)^{N}$ define $u_{h}^{y} \in \ell^{p}\left(h \mathbb{Z}^{N}\right)$ by $u_{h}^{y}(x)=u(y+x)$ for any $x \in h \mathbb{Z}^{N}$. We compute the mean of $F_{h}\left(u_{h}^{y}\right)$ over $(0, h)^{N}$ :

$$
\begin{aligned}
h^{-N} \int_{(0, h)^{N}} F_{h}\left(u_{h}^{y}\right) d y & =\sum_{\xi \in \mathbb{Z}^{N}} \phi(\xi) \sum_{x \in h \mathbb{Z}^{N}} \int_{(0, h)^{N}} \frac{1}{h} f_{\xi}\left(\frac{(u(y+x)-u(y+x+h \xi))^{2}}{h}\right) d y \\
& =\sum_{\xi \in \mathbb{Z}^{N}} \phi(\xi) \int_{\mathbb{R}^{N}} \frac{1}{h} f_{\xi}\left(\frac{(u(y)-u(y+h \xi))^{2}}{h}\right) d y .
\end{aligned}
$$

At this point, we exactly follow Gobbino's proof, writing

$$
\begin{aligned}
\int_{\mathbb{R}^{N}} \frac{1}{h} f_{\xi}\left(\frac{(u(y)-u(y+h \xi))^{2}}{h}\right) d y & =\int_{\xi^{\perp}} d \mathcal{H}^{N-1}(z) \int_{\mathbb{R}} d t \frac{1}{h} f_{\xi}\left(\frac{\left(u\left(z+t \frac{\xi}{|\xi|}\right)-u\left(z+t \frac{\xi}{|\xi|}+h \xi\right)\right)^{2}}{h}\right) \\
& =|\xi| \int_{\xi^{\perp}} d \mathcal{H}^{N-1}(z) \int_{\mathbb{R}} d s \frac{1}{h} f_{\xi}\left(\frac{(u(z+s \xi)-u(z+(s+h) \xi))^{2}}{h}\right) \\
& =|\xi| \int_{\xi^{\perp}} d \mathcal{H}^{N-1}(z) F_{\xi, h}^{1}\left(u_{z, \xi}\right)
\end{aligned}
$$


where $u_{z, \xi}(s)=u(z+s \xi)$ and we have set

$$
F_{\xi, h}^{1}(v)=\int_{\mathbb{R}} \frac{1}{h} f_{\xi}\left(\frac{(v(s)-v(s+h))^{2}}{h}\right) d s
$$

for any measurable function $v$. Since we assumed $f_{\xi}(t) \leq \alpha_{\xi} t \wedge \beta_{\xi}$, we have

$$
F_{\xi, h}^{1}(v) \leq \int_{\mathbb{R}} \alpha_{\xi}\left|\frac{v(s)-v(s+h)}{h}\right|^{2} \wedge \frac{\beta_{\xi}}{h} d s
$$

and as shown in [21] by Gobbino, this is less than

$$
\alpha_{\xi} \int_{\mathbb{R}}\left|v^{\prime}(s)\right|^{2} d s+\beta_{\xi} \mathcal{H}^{0}\left(S_{v}\right)
$$

provided $v \in S B V_{l o c}(\mathbb{R})$ and this expression is finite. (To check this, just compute the integral separately over $S_{v}^{h}=\bigcup_{s \in S_{v}}[s-h, s]$ and over $\mathbb{R} \backslash S_{v}^{h}$.)

Therefore,

$$
\begin{aligned}
h^{-N} \int_{(0, h)^{N}} F_{h}\left(u_{h}^{y}\right) d y & \leq \sum_{\xi \in \mathbb{Z}^{N}} \phi(\xi)|\xi| \int_{\xi^{\perp}} d \mathcal{H}^{N-1}(z) F_{\xi, h}^{1}\left(u_{z, \xi}\right) \\
& \leq \sum_{\xi \in \mathbb{Z}^{N}} \phi(\xi)|\xi| \int_{\xi^{\perp}} d \mathcal{H}^{N-1}(z)\left\{\alpha_{\xi} \int_{\mathbb{R}}|\langle\nabla u(z+s \xi), \xi\rangle|^{2} d s+\beta_{\xi} \mathcal{H}^{0}\left(S_{u_{z, \xi}}\right)\right\} \\
& =\sum_{\xi \in \mathbb{Z}^{N}} \phi(\xi)\left\{\int_{\mathbb{R}^{N}} \alpha_{\xi}|\langle\nabla u(x), \xi\rangle|^{2} d x+\int_{S_{u}} \beta_{\xi}\left|\left\langle\nu_{u}(x), \xi\right\rangle\right| d \mathcal{H}^{N-1}(x)\right\}=F(u) .
\end{aligned}
$$

Thus, for $y$ in some set of positive measure in $(0, h)^{N}$,

$$
F_{h}\left(u_{h}^{y}\right) \leq F(u)
$$

For all $h$ we choose $y_{h}$ such that inequality (19) holds and set $u_{h}=u_{h}^{y_{h}}$. We check easily that if $u$ is continuous at $x \in \mathbb{R}^{N}$ then $u_{h}(x) \rightarrow u(x)$ as $h \downarrow 0$ (since $u_{h}(x)=u\left(x^{\prime}\right)$ for some $x^{\prime}$ such that $\left|x-x^{\prime}\right|<\frac{3}{2} \sqrt{N} h$ ). Since $u$ is almost everywhere continuous, $u_{h}$ converges to $u$ a.e. in $\mathbb{R}^{N}$. We also have $\left\|u_{h}\right\|_{L^{\infty}\left(\mathbb{R}^{N}\right)} \leq\|u\|_{L^{\infty}\left(\mathbb{R}^{N}\right)}$ and the functions $u_{h}, u$ are zero outside some compact set so that by Lebesgue's theorem $u_{h} \rightarrow u$ in $L^{p}\left(\mathbb{R}^{N}\right)$. Since clearly, (17) holds for this sequence $u_{h}$, the proof of the case $\Omega=\mathbb{R}^{N}$ is achieved.

We now return to the general case where $\Omega$ is a Lipschitz domain. The method we use in order to localize the previous result is adapted from [11]. We choose a function $u \in G S B V_{l o c}(\Omega) \cap L^{p}(\Omega)$, and once again invoking Lemma 2 we see that it is not restrictive to assume that $u$ is bounded with bounded support. Since we assumed that $\partial \Omega$ is Lipschitz, (and since $u$ is zero outside some bounded set) we can extend $u$ outside of $\Omega$ (using the same reflection procedure as for instance in [14] for the extension of $W^{1, p}$ functions) into a bounded compactly supported $S B V$ function (still denoted by $u$ ) such that $\mathcal{H}^{N-1}\left(\partial \Omega \cap S_{u}\right)=0$ and $F\left(u, \mathbb{R}^{N}\right)<+\infty$. Then, we build $\left(u_{h}\right)$ like previously, such that $u_{h}$ goes to $u$ in $L^{p}\left(\mathbb{R}^{N}\right)$ and

$$
\underset{h \downarrow 0}{\limsup } F_{h}\left(u_{h}, \mathbb{R}^{N}\right) \leq F\left(u, \mathbb{R}^{N}\right) .
$$

We can write

$$
F_{h}\left(u_{h}, \mathbb{R}^{N}\right) \geq F_{h}\left(u_{h}, \Omega\right)+F_{h}\left(u_{h}, \bar{\Omega}^{c}\right)
$$


where $\bar{\Omega}^{c}$ is the complement of $\bar{\Omega}$ in $\mathbb{R}^{N}$. Notice that we have dropped all terms involving differences of values of $u_{h}$ at one point in $\bar{\Omega}$ and another in $\Omega^{c}$. Sending $h$ to zero we get

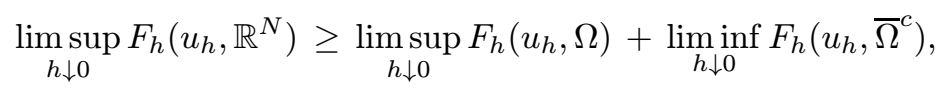

and we deduce from (8) that

$$
\underset{h \downarrow 0}{\limsup } F_{h}\left(u_{h}, \Omega\right)+F\left(u, \bar{\Omega}^{c}\right) \leq \limsup _{h \downarrow 0} F_{h}\left(u_{h}, \mathbb{R}^{N}\right) \leq F\left(u, \mathbb{R}^{N}\right) .
$$

Thus, $u$ being extended in such a way that $F\left(u, \bar{\Omega}^{c}\right)<+\infty$,

$$
\limsup _{h \downarrow 0} F_{h}\left(u_{h}, \Omega\right) \leq F(u, \bar{\Omega}) .
$$

Since $\mathcal{H}^{N-1}\left(\partial \Omega \cap S_{u}\right)=0, F(u, \bar{\Omega})=F(u, \Omega)$ and we get the thesis. This achieves the proof of Theorem 1 .

\subsection{Proof of Theorem 2}

For any $h>0$ let $\left(u_{h}\right)_{h>0}$ be a minimizer in $\ell^{p}\left(\Omega \cap h \mathbb{Z}^{N}\right)$ of

$$
F_{h}(u)+\int_{\Omega}|u(x)-g(x)|^{p} d x
$$

where $g \in L^{\infty}(\Omega) \cap L^{p}(\Omega)$.

Replacing $u_{h}$ with $\left(-\|g\|_{L^{\infty}(\Omega)} \vee u_{h}\right) \wedge\|g\|_{L^{\infty}(\Omega)}$ we decrease the energy, so that in fact $\left\|u_{h}\right\|_{L^{\infty}(\Omega)} \leq\|g\|_{L^{\infty}(\Omega)}$. In view of Lemma 1 , since $\sup _{h>0} F_{h}\left(u_{h}\right)<+\infty$, some subsequence $\left(u_{h_{j}}\right)_{j \geq 1}$ of $\left(u_{h}\right)_{h>0}$ converges to a function $u \in S B V_{l o c}(\Omega)$ a.e. in $\Omega$. From the uniform boundedness of the $u_{h}$ we deduce that $u_{h_{j}} \rightarrow u$ in $L_{l o c}^{p}(\Omega)$.

If $|\Omega|<+\infty$, the convergence is in $L^{p}(\Omega)$ and we simply conclude invoking Theorem 4 (Appendix B). Otherwise, we know (by the remark at the end of Section 3.2 and Fatou's lemma) that

$$
F(u)+\int_{\Omega}|u(x)-g(x)|^{p} d x \leq \liminf _{j \rightarrow \infty} F_{h_{j}}\left(u_{h_{j}}\right)+\int_{\Omega}\left|u_{h_{j}}(x)-g(x)\right|^{p} d x .
$$

For any $v \in L^{p}(\Omega)$, we consider $\left(v_{h_{j}}\right)_{j \geq 1}$ a sequence converging to $v$ in $L^{p}(\Omega)$ such that

$$
\limsup _{j \rightarrow \infty} F_{h_{j}}\left(v_{h_{j}}\right) \leq F(v) .
$$

For all $j$ we have that

$$
F_{h_{j}}\left(u_{h_{j}}\right)+\int_{\Omega}\left|u_{h_{j}}(x)-g(x)\right|^{p} d x \leq F_{h_{j}}\left(v_{h_{j}}\right)+\int_{\Omega}\left|v_{h_{j}}(x)-g(x)\right|^{p} d x,
$$

so that at the limit we get

$$
F(u)+\int_{\Omega}|u(x)-g(x)|^{p} d x \leq F(v)+\int_{\Omega}|v(x)-g(x)|^{p} d x,
$$

showing the minimality of $u$. If we choose $v=u$, we also deduce that

$$
\lim _{j \rightarrow \infty}\left\|u_{h_{j}}-g\right\|_{L^{p}(\Omega)}=\|u-g\|_{L^{p}(\Omega)},
$$


thus, by equi-integrability, $u_{h_{j}} \rightarrow u$ strongly in $L^{p}(\Omega)$, since we had the convergence in $L_{l o c}^{p}(\Omega)$. In the case where we minimize

$$
F_{h}(u)+\left(\left\|u-g^{h}\right\|_{p}\right)^{p}
$$

instead of (20) the proof is not different.

\section{Appendix A. Special Functions of Bounded VARiation}

\section{A.1. The spaces $S B V$ and $G S B V$ : definitions and main properties}

In this section we define shortly the "special functions with bounded variation" and state a few properties. See for instance [3] or [2] for further details. Given $\Omega \subseteq \mathbb{R}^{N}$ and $u: \Omega \rightarrow[-\infty,+\infty]$ a measurable function, we first define the approximate upper limit of $u$ at $x \in \Omega$ as

$$
u_{+}(x)=\inf \left\{t \in[-\infty,+\infty]: \lim _{\rho \downarrow 0} \frac{\left|\{y: u(y)>t\} \cap B_{\rho}(x)\right|}{\rho^{N}}=0\right\}
$$

where $B_{\rho}(x)$ is the ball of radius $\rho$ centered at $x$ and $|E|$ denotes the Lebesgue measure of the set $E$. The approximate lower limit $u_{-}(x)$ is defined in the same way $\left(i . e ., u_{-}(x)=-(-u)_{+}(x)\right)$. The set

$$
S_{u}=\left\{x \in \Omega: u_{-}(x)<u_{+}(x)\right\},
$$

is the set of essential discontinuities of $u$, it is a (Lebesgue-)negligible Borel set. If $x \notin S_{u}$, we say that $u$ is approximately continuous at $x$ and we write $\tilde{u}(x)=u_{-}(x)=u_{+}(x)=\operatorname{ap} \lim _{y \rightarrow x} u(y)$.

A function $u \in L^{1}(\Omega)$ is a function of bounded variation if its distributional derivative $D u$ is a vector-valued measure with finite total variation in $\Omega$ (equivalently, if the partial distributional derivatives $D_{i} u, i=1, \ldots, N$, are real-valued measures with finite total variation in $\Omega$ ). The space of functions of bounded variation is denoted by $B V(\Omega)$. For the general theory we refer to $[14,15,20,24]$. If $u \in B V(\Omega)$, the set $S_{u}$ is countably $\left(\mathcal{H}^{N-1}, N-1\right)$-rectifiable, i.e,

$$
S_{u}=\bigcup_{i=1}^{\infty} K_{i} \cup \mathcal{N}
$$

where $\mathcal{H}^{N-1}(\mathcal{N})=0$ and each $K_{i}$ is a compact subset of a $C^{1}$-hypersurface $\Gamma_{i}$. There exists a Borel function $\nu_{u}: S_{u} \rightarrow \mathbb{S}^{N-1}$ such that $\mathcal{H}^{N-1}$-a.e. in $S_{u}$ the vector $\nu_{u}(x)$ is normal to $S_{u}$ at $x$ in the sense that it is normal to $\Gamma_{i}$ if $x \in K_{i}$. For every $u, v \in B V(\Omega)$, we must therefore have $\nu_{u}= \pm \nu_{v} \mathcal{H}^{N-1}$-a.e. in $S_{u} \cap S_{v}$.

For every $u \in B V(\Omega)$ the measure $D u$ can be decomposed as follows:

$$
D u=\nabla u(x) d x+\left(u_{+}-u_{-}\right) \nu_{u} \mathcal{H}^{N-1}\left\llcorner S_{u}+C u\right.
$$

where $\nabla u$ is the approximate gradient of $u$, defined a.e. in $\Omega$ by

$$
\text { ap } \lim _{y \rightarrow x} \frac{u(y)-u(x)-\langle\nabla u(x), y-x\rangle}{|y-x|}=0,
$$

$\mathcal{H}^{N-1}\left\llcorner S_{u}\right.$ is the restriction of the $N-1$ dimensional Hausdorff measure to the set $S_{u}$, and $C u$ is the Cantor part of the measure $D u$, which is singular with respect to the Lebesgue measure and such that $|C u|(E)=0$ for any $E$ with $\mathcal{H}^{N-1}(E)<+\infty$.

We say that a function $u \in B V(\Omega)$ is a special function with bounded variation if $C u=0$, which means that the singular part of the distributional derivative $D u$ is concentrated on the jump set $S_{u}$. We denote by 
$S B V(\Omega)$ the space of such functions. We also define the space $G S B V(\Omega)$ of generalized $S B V$ functions as the set of all measurable functions $u: \Omega \rightarrow[-\infty,+\infty]$ such that for any $k>0, u^{k}=(-k \vee u) \wedge k \in S B V(\Omega)$ (where $X \wedge Y=\min (X, Y)$ and $X \vee Y=\max (X, Y))$, following Ambrosio's definition in [1].

If $u \in G S B V_{l o c}(\Omega) \cap L_{l o c}^{1}(\Omega), u$ has an approximate gradient a.e. in $\Omega$, moreover, as $k \uparrow \infty$,

$$
\begin{gathered}
\nabla u^{k} \rightarrow \nabla u \text { a.e. in } \Omega, \quad \text { and } \quad\left|\nabla u^{k}\right| \uparrow|\nabla u| \text { a.e. in } \Omega ; \\
S_{u^{k}} \subseteq S_{u}, \quad \mathcal{H}^{N-1}\left(S_{u^{k}}\right) \rightarrow \mathcal{H}^{N-1}\left(S_{u}\right) \quad \text { and } \quad \nu_{u^{k}}=\nu_{u} \quad \mathcal{H}^{N-1} \text {-a.e. in } S_{u^{k}} .
\end{gathered}
$$

Slicing. We consider now for $\xi \in \mathbb{S}^{N-1}$ the sets $\xi^{\perp}=\left\{x \in \mathbb{R}^{N}:\langle\xi, x\rangle=0\right\}$ and for any $z \in \xi^{\perp}$, $\Omega_{z, \xi}=\{t \in \mathbb{R}: z+t \xi \in \Omega\}$. On $\Omega_{z, \xi}$ we define a function $u_{z, \xi}: \Omega_{z, \xi} \rightarrow[-\infty,+\infty]$ by $u_{z, \xi}(s)=u(z+s \xi)$. If $u \in B V(\Omega)$, we have the following classical representation (see for instance [1,4]): for $\mathcal{H}^{N-1}$-a.e. $z \in \xi^{\perp}$, $u_{z, \xi} \in B V\left(\Omega_{z, \xi}\right)$ and for any Borel set $B \subseteq \Omega$

$$
\langle D u, \xi\rangle(B)=\int_{\xi^{\perp}} d \mathcal{H}^{N-1}(z) D u_{z, \xi}\left(B_{z, \xi}\right)
$$

where $B_{z, \xi}$ is defined in the same way as $\Omega_{z, \xi}$; conversely if $u_{z, \xi} \in B V\left(\Omega_{z, \xi}\right)$ for at least $N$ independent vectors $\xi \in \mathbb{S}^{N-1}$ and $\mathcal{H}^{N-1}$-a.e. $z \in \xi^{\perp}$, and if

$$
\int_{\xi^{\perp}} d \mathcal{H}^{N-1}(z)\left|D u_{z, \xi}\right|\left(\Omega_{z, \xi}\right)<+\infty
$$

then $u \in B V(\Omega)$. Now (see $[1,2]$ ), if $u \in S B V_{l o c}(\Omega)$, then for almost every $z \in \xi^{\perp}, u_{z, \xi} \in S B V_{l o c}\left(\Omega_{z, \xi}\right)$ (the converse is true provided this property is satisfied for at least $N$ independent vectors $\xi$ and $u$ has locally bounded variation), and the approximate derivative satisfies

$$
u_{z, \xi}^{\prime}(s)=\langle\nabla u(z+s \xi), \xi\rangle
$$

for a.e. $s \in \Omega_{z, \xi}$, moreover

$$
S_{u_{z, \xi}}=\left\{s \in \Omega_{z, \xi}: z+s \xi \in S_{u}\right\}
$$

and for any Borel set $B \subseteq \Omega$

$$
\int_{\xi^{\perp}} d \mathcal{H}^{N-1}(z) \mathcal{H}^{0}\left(B_{z, \xi} \cap S_{u_{z, \xi}}\right)=\int_{B}\left|\left\langle\nu_{u}(x), \xi\right\rangle\right| d \mathcal{H}^{N-1}(x) .
$$

Compactness. Eventually, we mention the following compactness and lower semi-continuity result that is proved in [1] (see also $[2,3]$ ):

Theorem 3 (Ambrosio). Let $\Omega$ be an open subset of $\mathbb{R}^{N}$ and let $\left(u_{j}\right)$ be a sequence in $G S B V(\Omega)$. Suppose that there exist $p \in[1, \infty]$ and a constant $C$ such that

$$
\int_{\Omega}\left|\nabla u_{j}\right|^{2} d x+\mathcal{H}^{N-1}\left(S_{u_{j}}\right)+\left\|u_{j}\right\|_{L^{p}(\Omega)} \leq C<+\infty
$$


for every $j$. Then there exist a subsequence (not relabelled) and a function $u \in G S B V(\Omega) \cap L^{p}(\Omega)$ such that

$$
\begin{aligned}
& u_{j}(x) \rightarrow u(x) \text { a.e. in } \Omega, \\
& \nabla u_{j} \rightarrow \nabla u \text { weakly in } L^{2}\left(\Omega, \mathbb{R}^{N}\right), \\
& \mathcal{H}^{N-1}\left(S_{u}\right) \leq \liminf _{j \rightarrow \infty} \mathcal{H}^{N-1}\left(S_{u_{j}}\right) .
\end{aligned}
$$

Moreover

$$
\int_{S_{u}}\left|\left\langle\nu_{u}, \xi\right\rangle\right| d \mathcal{H}^{N-1} \leq \liminf _{j \rightarrow \infty} \int_{S_{u_{j}}}\left|\left\langle\nu_{u_{j}}, \xi\right\rangle\right| d \mathcal{H}^{N-1}
$$

for every $\xi \in \mathbb{S}^{N-1}$.

Remark. In many works (see for instance [3]), according to De Giorgi's original definition, the space $G S B V(\Omega)$ is the larger space (here denoted by $G S B V_{l o c}(\Omega)$ ) of functions $u$ such that for every $k>0$ the truncated $u^{k}$ belongs to $S B V(A)$ for any open set $A \subset \subset \Omega$, i.e. such that $\bar{A}$ is compact and contained in $\Omega$. We preferred here to specify explicitly when we localize in the space variable by means of the notation $G S B V_{l o c}$. Notice that by a standard diagonalization technique Theorem 3 extends easily to the case where $u_{j}$ and $u$ are in $G S B V_{l o c}(\Omega)$.

\section{A.2. An application: the Mumford-Shah functional}

The functional originally introduced by Mumford and Shah, in order to modelize the image segmentation problem in a continuous setting, is the following

$$
\mathcal{G}(u, K)=\int_{\Omega \backslash K}|\nabla u(x)|^{2} d x+\mathcal{H}^{N-1}(K)+\int_{\Omega}|u(x)-g(x)|^{2} d x,
$$

where $g \in L^{\infty}(\Omega)$ is a given "original image", $K$ is a closed set and $u \in C^{1}(\Omega \backslash K)$. Ambrosio and De Giorgi introduced the weak formulation in $G S B V_{l o c}(\Omega)$

$$
G(u)=\int_{\Omega}|\nabla u(x)|^{2} d x+\mathcal{H}^{N-1}\left(S_{u}\right)+\int_{\Omega}|u(x)-g(x)|^{2} d x,
$$

and proved the existence of a minimizer for $G$ using Theorem 3. Then, De Giorgi, Carriero and Leaci established the existence of a minimizer for $\mathcal{G}$ by proving that if $u$ minimizes $G$, then $\mathcal{H}^{N-1}\left(\Omega \cap \overline{S_{u}} \backslash S_{u}\right)=0$ and $u \in C^{1}\left(\Omega \backslash \overline{S_{u}}\right)$, so that $\left(u, \overline{S_{u}}\right)$ minimizes $\mathcal{G}[13]$.

Adapting the proofs in [13], we can prove the following lemma ( $F$ is given by equation $(2))$ :

Lemma 3. Let $g \in L^{p}(\Omega) \cap L^{\infty}(\Omega)$ and consider the minimum problem

$$
\min _{u \in G S B V_{l o c}(\Omega)} F(u)+\int_{\Omega}|u(x)-g(x)|^{p} d x .
$$

Then:

- problem (23) has a solution;

- if $u \in G S B V_{\text {loc }}(\Omega)$ solves (23), then $\mathcal{H}^{N-1}\left(\Omega \cap \overline{S_{u}} \backslash S_{u}\right)=0$ and $u \in C^{1}\left(\Omega \backslash \overline{S_{u}}\right)$.

The existence of a solution comes from a direct application of Theorem 3, once we have noticed that we may consider minimizing sequences uniformly bounded in $L^{\infty}(\Omega)$ by $\|g\|_{L^{\infty}(\Omega)}$ (so that in fact the solution $u$ is in $\left.S B V_{l o c}(\Omega)\right)$. To get the semicontinuity of the surface term

$$
\int_{S_{u}} \sum_{\xi \in \mathbb{Z}^{N}} \phi(\xi) \beta_{\xi}\left|\left\langle\nu_{u}(x), \xi\right\rangle\right| d \mathcal{H}^{N-1}(x)=\sum_{\xi \in \mathbb{Z}^{N}} \phi(\xi) \beta_{\xi} \int_{S_{u}}\left|\left\langle\nu_{u}(x), \xi\right\rangle\right| d \mathcal{H}^{N-1}(x)
$$


we use the last semicontinuity inequality in Theorem 3 and Fatou's lemma.

For the second part of the lemma, we notice that $F$ may be written in the following way

$$
F(u)=\int_{\Omega} Q(\nabla u(x)) d x+\int_{S_{u}} N\left(\nu_{u}(x)\right) d \mathcal{H}^{N-1}(x),
$$

where for any $X \in \mathbb{R}^{N}$,

$$
Q(X)=\sum_{\xi \in \mathbb{Z}^{N}} \alpha_{\xi} \phi(\xi)|\langle X, \xi\rangle|^{2}
$$

and

$$
N(X)=\sum_{\xi \in \mathbb{Z}^{N}} \beta_{\xi} \phi(\xi)|\langle X, \xi\rangle|
$$

Since $\phi\left(e_{i}\right)>0$ for $i=1, \ldots, N, Q$ is a positive definite quadratic form and $N$ is a norm in $\mathbb{R}^{N}$, and there exist $\gamma, \gamma^{\prime}>0$ such that for any $u \in S B V_{l o c}(\Omega)$ and any Borel set $B$,

$$
\gamma \mathcal{H}^{N-1}\left(B \cap S_{u}\right) \leq \int_{B \cap S_{u}} N\left(\nu_{u}(x)\right) d \mathcal{H}^{N-1}(x) \leq \gamma^{\prime} \mathcal{H}^{N-1}\left(B \cap S_{u}\right) .
$$

This property is sufficient to adapt the proof in [13] to this particular problem.

\section{Appendix B. The $\Gamma$-Convergence}

We shortly define the $\Gamma$-convergence of functionals (in metric spaces) and its main properties. For more details we refer to [12].

Given a metric space $(X, d)$ and $F_{k}: X \rightarrow[-\infty,+\infty]$ a sequence of functions, we define for every $u \in X$ the $\Gamma$-liminf of $F$

$$
F^{\prime}(u)=\Gamma-\liminf _{k \rightarrow \infty} F_{k}(u)=\inf _{u_{k} \rightarrow u} \liminf _{k \rightarrow \infty} F_{k}\left(u_{k}\right)
$$

and the $\Gamma$-limsup of $F$

$$
F^{\prime \prime}(u)=\Gamma-\limsup _{k \rightarrow \infty} F_{k}(u)=\inf _{u_{k} \rightarrow u} \limsup _{k \rightarrow \infty} F_{k}\left(u_{k}\right),
$$

and we say that $F_{k} \Gamma$-converges to $F: X \rightarrow[-\infty,+\infty]$ if $F^{\prime}=F^{\prime \prime}=F . F^{\prime}, F^{\prime \prime}$, and $F$ (if it exists) are lower semi-continuous on $X$. We have the following two properties:

1. $F_{k} \Gamma$-converges to $F$ if and only if for every $u \in X$,

(i) for every sequence $u_{k}$ converging to $u, F(u) \leq \liminf _{k \rightarrow \infty} F_{k}\left(u_{k}\right)$;

(ii) there exists a sequence $u_{k}$ that converges to $u$ and such that $\lim \sup _{k \rightarrow \infty} F_{k}\left(u_{k}\right) \leq F(u)$;

2. If $G: X \rightarrow \mathbb{R}$ is continuous and $F_{k} \Gamma$-converges to $F$, then $F_{k}+G \Gamma$-converges to $F+G$.

The following result makes clear the interest of the notion of $\Gamma$-convergence:

Theorem 4. Assume $F_{k} \Gamma$-converges to $F$ and for every $k$ let $u_{k}$ be a minimizer of $F_{k}$ over $X$. Then, if the sequence (or a subsequence) $u_{k}$ converges to some $u \in X, u$ is a minimizer for $F$ and $F_{k}\left(u_{k}\right)$ converges to $F(u)$.

Eventually, we give the following definition of $\Gamma$-convergence in the case where $\left(F_{h}\right)_{h>0}$ is a family of functionals on $X$ indexed by a continuous parameter $h$ : we say that $F_{h} \Gamma$-converges to $F$ in $X$ as $h \downarrow 0$ if and only if for every sequence $\left(h_{j}\right)$ that converges to zero as $j \rightarrow \infty, F_{h_{j}} \Gamma$-converges to $F$. 


\section{REFERENCES}

[1] L. Ambrosio. A compactness theorem for a new class of functions with bounded variation. Boll. Un. Mat. Ital. (7) 3 (1989) $857-881$.

[2] L. Ambrosio. Variational problems in SBV and image segmentation. Acta Appl. Math. 17 (1989) 1-40.

[3] L. Ambrosio. Existence theory for a new class of variational problems. Arc. Rational Mech. Anal. 111 (1990) $291-322$.

[4] L. Ambrosio and V.M. Tortorelli. Approximation of functionals depending on jumps by elliptic functionals via $\Gamma$-convergence. Comm. Pure Appl. Math. 43 (1990) 999-1036.

[5] L. Ambrosio and V.M. Tortorelli. On the approximation of free discontinuity problems. Boll. Un. Mat. Ital. (7) 6 (1992) 105-123.

[6] G. Aubert, M. Barlaud, P. Charbonnier and L. Blanc-Féraud. Deterministic edge-preserving regularization in computed imaging. Technical report TR\#94-01, I3S, CNRS URA 1376, Sophia-Antipolis, France (1994).

[7] A. Blake and A. Zisserman. Visual Reconstruction. MIT Press (1987).

[8] B. Bourdin and A. Chambolle. Implementation of a finite-elements approximation of the Mumford-Shah functional. Technical report 9844, Ceremade, University of Paris-Dauphine, 1998; preprint LPMTM, University of Paris-Nord, 1998; Numer. Math. (to appear).

[9] A. Chambolle. Un théorème de $\Gamma$-convergence pour la segmentation des signaux. C. R. Acad. Sci. Paris 314 (1992) $191-196$.

[10] A. Chambolle. Image segmentation by variational methods: Mumford and Shah functional and the discrete approximations. SIAM J. Appl. Math. 55 (1995) 827-863.

[11] A. Chambolle and G. Dal Maso. Discrete approximation of the Mumford-Shah functional in dimension two. Technical Report 9820, Ceremade, University of Paris-Dauphine, 1998; preprint SISSA 29/98/M, Trieste; RAIRO Modél. Math. Anal. Numér. (to appear).

[12] G. Dal Maso. An introduction to $\Gamma$-convergence. Birkhäuser, Boston (1993).

[13] E. De Giorgi, M. Carriero and A. Leaci. Existence theorem for a minimum problem with free discontinuity set. Arch. Rational Mech. Anal. 108 (1989) 195-218.

[14] L.C. Evans and R.F. Gariepy. Measure Theory and Fine Properties of Functions. CRC Press, Boca Raton (1992).

[15] H. Federer. Geometric Measure Theory. Springer-Verlag, New-York (1969).

[16] D. Geiger and F. Girosi. Parallel and deterministic algorithms for MRFs: surface reconstruction. IEEE Trans. PAMI 13 (1991) 401-412.

[17] D. Geiger and A. Yuille. A common framework for image segmentation. Internat. J. Comput. Vision 6 (1991) $227-243$.

[18] D. Geman and G. Reynolds. Constrained image restoration and the recovery of discontinuities. IEEE Trans. PAMI 3 (1992) $367-383$.

[19] S. Geman and D. Geman. Stochastic relaxation, Gibbs distributions, and the Bayesian restoration of images. IEEE Trans. PAMI 6 (1984).

[20] E. Giusti. Minimal surfaces and functions of bounded variation. Birkhäuser, Boston (1984).

[21] M. Gobbino. Finite difference approximation of the Mumford-Shah functional. Comm. Pure Appl. Math. 51 (1998) $197-228$.

[22] D. Mumford and J. Shah. Optimal approximation by piecewise smooth functions and associated variational problems. Comm. Pure Appl. Math. 42 (1989) 577-685.

[23] C.R. Vogel and M.E. Oman. Iterative methods for total variation denoising, in Proceedings of the Colorado Conference on Iterative Methods (1994).

[24] W.P. Ziemer. Weakly Differentiable Functions. Springer-Verlag, Berlin (1989). 